\title{
ID01 inhibition potentiates vaccine-induced immunity against pancreatic adenocarcinoma
}

\author{
Alex B. Blair, ${ }^{1,2,3,4}$ Jennifer Kleponis, ${ }^{1,2}$ Dwayne L. Thomas II, ${ }^{1,2,4}$ Stephen T. Muth, ${ }^{1,2,4}$ Adrian G. Murphy, ${ }^{1,2,4}$ Victoria Kim, ${ }^{1,2,3}$ \\ and Lei Zheng , $^{1,2,3,4}$ \\ 'The Sidney Kimmel Comprehensive Cancer Center, ${ }^{2}$ Department of Oncology, ${ }^{3}$ Department of Surgery, and ${ }^{4}$ The Pancreatic Cancer Precision Medicine Center of Excellence Program, The Johns Hopkins \\ University School of Medicine, Baltimore, Maryland, USA.
}

\begin{abstract}
Pancreatic ductal adenocarcinoma (PDAC) represents an immune quiescent tumor that is resistant to immune checkpoint inhibitors. Previously, our group has shown that a GM-CSF-secreting allogenic pancreatic tumor cell vaccine (GVAX) may prime the tumor microenvironment by inducing intratumoral T cell infiltration. Here, we show that untreated PDACs express minimal indoleamine-2,3-dioxygenase (ID01); however, GVAX therapy induced IDO1 expression on tumor epithelia as well as vaccine-induced tertiary lymphoid aggregates. ID01 expression plays a role in regulating the polarization of Th1, Th17, and possibly T regulatory cells in PDAC tumors. ID01 inhibitor enhanced antitumor efficacy of GVAX in a murine model of PDACs. The combination of vaccine and IDO1 inhibitor enhanced intratumoral T cell infiltration and function, but adding anti-PD-L1 antibody to the combination did not offer further synergy and in fact may have had a negative interaction, decreasing the number of intratumoral effector T cells. Additionally, IDO1 inhibitor in the presence of vaccine therapy did not significantly modulate intratumoral myeloid-derived suppressor cells quantitatively, but diminished their suppressive effect on CD8 ${ }^{+}$proliferation. Our study supports the combination of IDO1 inhibitor and vaccine therapy; however, it does not support the combination of ID01 inhibitor and anti-PD-1/PD-L1 antibody for T cell-inflamed tumors such as PDACs treated with vaccine therapy.
\end{abstract}

\section{Introduction}

Pancreatic adenocarcinoma (PDAC) remains one of the most aggressive malignancies due to the overall ineffectiveness and lack of durable responses with contemporary systemic therapies. This has led to the urgent necessity for development of novel therapeutic approaches such as immunotherapy. Unfortunately, PDAC represents an immune quiescent, so-called "cold" tumor that is not sensitive to immune checkpoint inhibitors (1). Therefore, PDAC also serves as an ideal model for studying how to overcome the resistance to immune checkpoint inhibitor treatment (2-4).

Previously, our group showed that vaccine therapy induces $\mathrm{T}$ cell infiltration into tumors, subsequently priming the tumor microenvironment with these $\mathrm{T}$ cells. We showed that tertiary lymphoid aggregates were formed in PDACs resected from patients who received a GM-CSF-secreting, allogeneic pancreatic tumor whole-cell vaccine (GVAX) 2 weeks prior to surgical resection (5). For the first time, we showed that vaccine therapy can possibly convert a cold tumor to a T cell-inflamed hot tumor. However, this vaccine priming process is only the very first step to elicit an

Authorship note: $A B B, J K$, and DLT contributed equally to this work. Conflict of interest: $L Z$ receives grant supports from Bristol-Meyer Squibb, Merck, iTeos, Amgen, Gradalis, and Halozyme; consults for Astrozeneca, Merck, Oncorus, Alphamab, Sound Biologics, Biosion, Fosun Biopharmaceutical, Foundation Medicine, and Mingruizhiyao; is a shareholder of Alphamab and Mingruizhiyao; and receives the royalty for licensing GVAX to Aduro Biotech.

Copyright: () 2019 American Society for Clinical Investigation

Submitted: August 7, 2018; Accepted: February 5, 2019.

Reference information: / Clin Invest. 2019;129(4):1742-1755.

https://doi.org/10.1172/JCl124077. antitumor immune response. The immune checkpoint signaling cascade is also induced by vaccine therapy $(6,7)$. Therefore, we hypothesize that vaccine therapy may have primed PDACs for the treatment of previously ineffective immune checkpoint inhibitors. We confirmed this hypothesis in preclinical studies and are now testing this concept in several clinical trials (8).

The immune checkpoint signaling induced by vaccine therapy is more complicated than solely the PD-L1/PD-1 signaling pathway. We found that higher indoleamine-2,3-dioxygenase (IDO1) expression in the tertiary lymphoid aggregates is correlated with poorer survival following GVAX treatment (5). IDO1 is encoded by the IDO1 gene and is an intracellular enzyme that is involved in the rate-limiting step of the catabolism of L-tryptophan, an important regulator of metabolism. Tryptophan starvation and the influx of downstream catabolites such as kynurenine suppress the activation of T cells and NK cells while enhancing Treg differentiation and immunosuppression (9-11). Tumor cells transfected with IDO1 were not rejected in mice treated with a vaccine therapy (12). Furthermore, IDO1 deficiency resulted in decreased immune escape in a preclinical model of lung cancer (13). IDO1 expression has been found in a variety of cancers, including PDAC. Expression of IDO1 is upregulated by metastatic PDAC cells as a mechanism of immunologic evasion (14). IDO1 expression is also reported in regulatory dendritic cells (DCs) and is prompted by an autocrine interferon process controlled by CTLA-4 pathway receptors on regulatory T cells (Treg) (15). This expression of IDO1 subsequently converts the DCs into a more quiescent state and reduces their antigen presenting capacity to T cells. Moreover, IDO-expressing DCs are also able to drive the differentiation of $\mathrm{T}$ helper cells to 
Tregs, further suppressing an antitumor immune response (16). In addition to direct inhibitory effects of IDO1 tumor expression on $\mathrm{T}$ cell activity, myeloid-derived suppressor cells (MDSCs) are another cell subtype relevant to the IDO1 pathway (17). MDSC functions, including the suppression of antitumor immune responses and $\mathrm{CD}^{+} \mathrm{T}$ cell proliferation, are thought to be regulated through IDO1 pathways (17-19).

There are several small-molecule inhibitors of IDO1 in clinical testing. The most tested one is epacadostat (INCB024360), which is currently the focus of several clinical trials encompassing multiple tumor types. It is an orally available hydroxyamidine small-molecule inhibitor that potently and selectively inhibits IDO1. The phase I dose-escalation study of epacadostat included 52 patients with multiple tumor types, including colorectal cancer and melanoma. There was no maximum tolerated dose identified and no objective responses were reported, although 15 patients (28\%) had stable disease at 56 days. Doses of $300 \mathrm{mg}$ or greater BID achieved greater than $90 \%$ inhibition of IDO1 throughout the dosing period (20). Epacadostat was combined with the anti-CTLA4 antibody ipilimumab in patients with advanced melanoma in a phase I/II study. Preliminary data from this study (NCT01604889) showed that epacadostat combined with ipilimumab resulted in clinically significant ALT elevations after 30-76 days of treatment but were reversible with steroids and treatment discontinuation. In 6 of 8 patients, there were tumor reductions by the time of first imaging. These preliminary data suggest the role of IDO1 inhibition in providing enhanced antitumor effects with anti-CTLA4 therapy (21). Subsequently, epacadostat was found to be safer in combination with pembrolizumab, an anti-PD-1 antibody, in a phase I/II Echo-202/Keynote-037 clinical trial of multiple tumor types (NCT02178722); the combination showed $56 \%$ response rate in 54 advanced melanomas (22). Nevertheless, the follow-up phase III Keynote-252/Echo-301 clinical trial (NCT02752074) failed to demonstrate that the combination of epacadostat and pembrolizumab is superior over the single-agent pembrolizumab as the first-line therapy of advanced melanoma (23). Notably, little IDO1 expression is appreciated in untreated PDAC, thus the use of IDO1 inhibitor as a single agent is not effective for this disease.

While INCBO24360 was being tested in clinical studies, we independently found that, as anticipated, IDO1 expression is induced in the tumor epithelia of PDACs treated by GVAX and lymphoid aggregates induced by GVAX due to the known regulation of IDO1 expression by IFN- $\gamma$ and other inflammatory cytokines $(24,25)$. Thus, we tested the hypothesis that vaccine therapy can prime PDACs for IDO1 inhibitor treatment by inducing expression in a preclinical model of PDACs. In this study, we showed that EOS200271, a selective, small-molecule inhibitor of IDO1, which was tested in a phase I dose-escalation study (NCT02764151) in patients with malignant gliomas, was able to markedly enhance the antitumor activity of GVAX in a preclinical model of PDAC through downstream regulation of $\mathrm{CD} 8^{+}$cell effector function and $\mathrm{CD}^{+}$cell polarization. We also found that the addition of antiPD-1/PD-L1 antibody would only modestly enhance the antitumor activity of the combination of IDO1 inhibitor and GVAX and that combining anti-PD-1/PD-L1 antibody with IDO1 inhibitor and GVAX did not provide additional synergistic immune effect, instead observing a reduction of effector $\mathrm{T}$ cell infiltration and tumor-specific cytotoxic $\mathrm{T}$ cell activity compared with combination without anti-PD-1/PD-L1 antibodies.

\section{Results}

Untreated PDACs express minimal IDO1, and anticancer treatments induce IDO1 expression in PDACs. To understand whether an immune quiescent neoplasm such as PDAC expresses IDO1, we performed immunohistochemistry (IHC) of IDO1 on PDACs surgically resected from patients untreated with preoperative chemotherapy or radiation therapy $(n=21)$. We found that none of the PDAC specimens expressed IDO1 on the tumor epithelium and that the expression of IDO1 on the nonepithelial cells was also scarce (Figure 1, A and B). Then, we examined whether cancer vaccine therapy can induce IDO1 expression in PDACs. To this end, we performed IHC on PDACs surgically resected from patients who were treated with pancreatic cancer GVAX 2 weeks prior to surgical resection $(n=21)$. As shown in Figure 1B, 28.5\% of PDACs following GVAX treatment expressed IDO1 strongly on the tumor epithelium (IDO1 ${ }^{\text {hi) }}$. The remaining PDACs did not express IDO1 or only expressed IDO1 modestly. Of note, high expression of IDO1 in GVAX-treated PDAC was significantly associated with inferior patient survival according to a previously conducted microarray analysis (Figure 1C) (5). The tumor epithelia with strong expression of IDO1 were accompanied by an abundant infiltration of $\mathrm{CD}^{+}$cells and $\mathrm{CD}^{+}$lymphocytes (Figure 1D). By contrast, the tumor epithelia with weak or absent expression of IDO1 (IDO1 ${ }^{10}$ ) were accompanied by few observed $\mathrm{CD}^{+}$and $\mathrm{CD}^{+}$cells. This is consistent with the notion that IDO1 expression is induced by the adaptive immune response $(24,25)$. However, the infiltration of $\mathrm{CD}^{+}$cells appeared to be similar regardless of the IDO1 expression. In addition, we found that IDO1 expression on the tumor epithelia was induced in 4 of 20 PDACs following neoadjuvant chemotherapy and radiation therapy (Supplemental Figure 1; supplemental material available online with this article; https://doi.org/10.1172/JCI124077DS1). In summary, anticancer treatments, including vaccine therapy and chemotherapy/radiation therapy, may induce IDO1 expression in PDACs, and higher IDO1 expression is associated with inferior overall survival (OS).

PD-L1 expression and IDO1 expression are overlapped in vaccine-induced lymphoid aggregates. Next, we examined IDO1 expression in the previously shown GVAX-induced tertiary lymphoid aggregates. There were significantly more $\mathrm{IDO}^{+}$cells in the GVAX-induced intratumoral lymphoid aggregates than those in the peritumoral lymphoid aggregates in either vaccinated PDACs or unvaccinated PDACs (Figure 1E). Essentially, in all of the vaccinated PDACs at least one intratumoral lymphoid aggregate with $\mathrm{IDO}^{+}$cells could be identified. Nevertheless, not all of the intratumoral lymphoid aggregates contained IDO1+ cells. Interestingly, when we stained IDO1 on cases that had been previously analyzed for PD-L1 expression, we found that if the lymphoid aggregates expressed PD-L1 they also expressed IDO1. However, among the lymphoid aggregates that expressed IDO1, not all expressed PD-L1 (Figure 1F). This result suggested that IDO1 expression and PD-L1 expression are overlapped in GVAX-induced lymphoid aggregates.

The combination of IDO1 inhibitor and the GVAX vaccine has potent antitumor activity in the preclinical model of PDACs. Previ- 

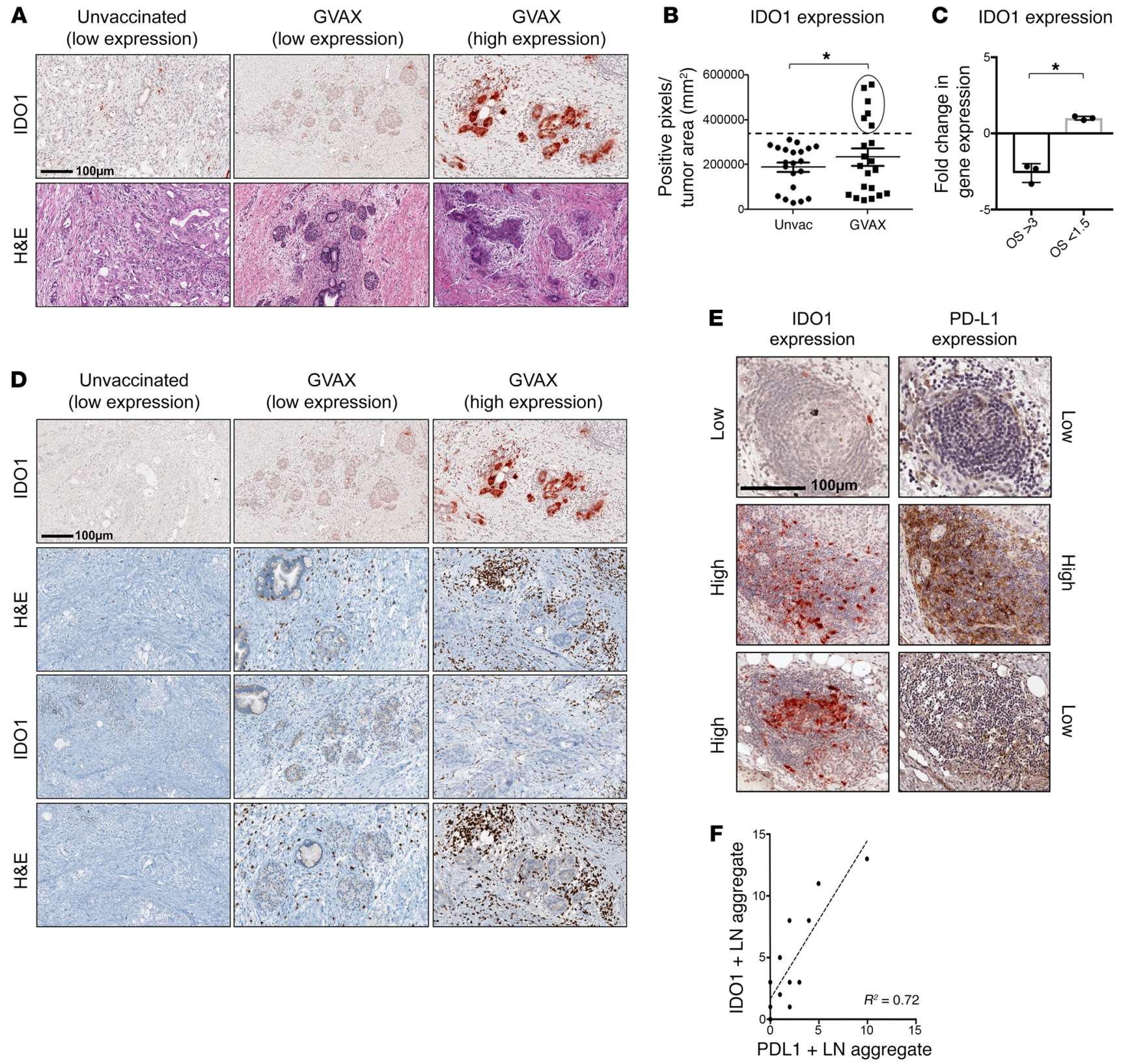

Figure 1. Pancreatic cancer treated with GVAX primes the tumor, upregulating IDO1 and PD-L1 pathway expression. (A) IHC staining of IDO1 expression in PDACs from patients treated with $(n=21)$ and without $(n=21)$ GVAX therapy. (B) Analysis of IDO1 IHC expression of total tumor area in PDACs treated with GVAX compared with untreated tumors, with an identified subset expressing ID01 highly in the GVAX-treated group. (C) Gene expression of IDO1 measured by microarray was compared between microdissected lymphoid aggregates from 16 tumors grouped according to overall patient survival (OS $>3$ years vs. < 1.5 years). (D) IHC staining of IDO1 expression and immune cell infiltration of $\mathrm{CD}^{+}, \mathrm{CD} 4^{+}$, and $\mathrm{CD} 8^{+}$cells in PDACs treated with and without GVAX therapy. The same representative sample in $\mathbf{A}$ is utilized again here. (E) IHC representations and (F) graphical comparison of ID01 and PD-L1 lymphoid aggregate expression by IHC following GVAX treatment $(n=16)$. If IDO1 expression is not upregulated, PD-L1 expression is not detectable. If PD-L1 expression is upregulated, IDO1 expression is upregulated. In some cases, ID01 expression is increased but PD-L1 expression remains low. ${ }^{*} P<0.05$ by unpaired $t$ test. Scale bars, $100 \mu \mathrm{m}$.

ously, we showed, in a preclinical model of PDAC, that PDACs are primed with PD-L1 expression and T cell infiltration by GVAX treatment and made sensitive to anti-PD-1 or PD-L1 therapies (8). Here we showed that GVAX can also prime IDO1 expression in PDACs, and thus we hypothesized that GVAX could similarly make PDACs sensitive to an IDO1 targeting therapy. We tested this hypothesis in syngeneic mice with diffuse liver metastases that were established by the hemispleen injection of murine
Panc02 PDAC cells and treatment with a small-molecule inhibitor of IDO1. As shown in Figure 2A, the IDO1 inhibitor alone did not have antitumor activity. However, when it was combined with GVAX treatment together with a low intravenous dose of cyclophosphamide (Cy/GVAX), it significantly improved the survival of tumor-bearing mice compared with Cy/GVAX alone. A single, low-dose Cy was administered to reduce Tregs. This result has further supported the concept that, to make PDACs sensitive to 
A

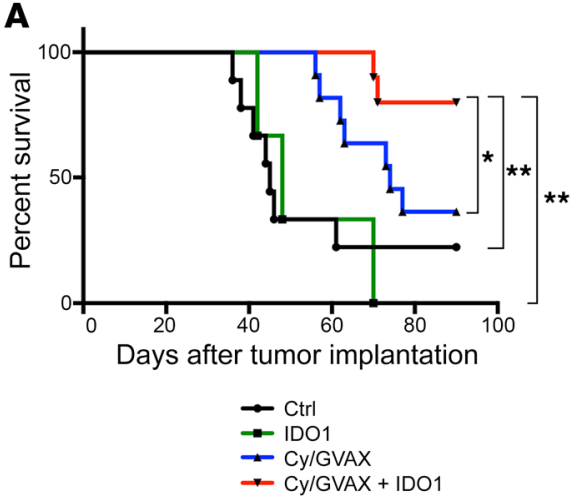

B

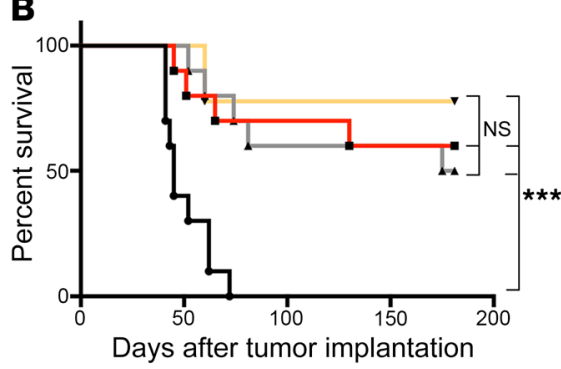

$$
\begin{aligned}
& \rightarrow \mathrm{Ctrl} \\
& \Rightarrow \mathrm{Cy} / \mathrm{GVAX}+\mathrm{IDO} 1 \\
& -\mathrm{Cy} / \mathrm{GVAX}+\mathrm{PD}-\mathrm{L} 1 \\
& \rightarrow \mathrm{Cy} / \mathrm{GVAX}+\text { IDO1 + PD-L1 }
\end{aligned}
$$
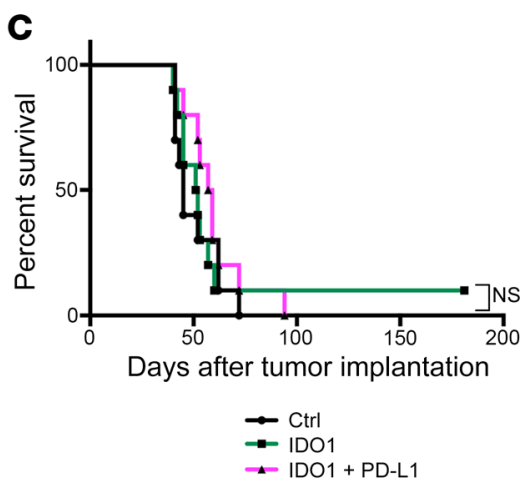

Figure 2. ID01 inhibitor in combination with irradiated whole-cell vaccine (GVAX) improves survival in a murine model of pancreatic cancer. Mice underwent hemispleen procedure receiving $2 \times 10^{6}$ Panc02 PDAC cells followed by administration of $100 \mathrm{mg} / \mathrm{kg}$ Cy on day 3 and GVAX on day 4 . IDO1 inhibitor $(200 \mu \mathrm{g} / \mathrm{kg})$ was administered by oral gavage twice a day starting on day 3 and continuing for 90 days. (A) Kaplan-Meier survival curves of mice treated with IDO1 inhibitor or Cy/GVAX alone and in combination. (B) Kaplan-Meier survival curves of mice with liver metastases formed by the Panc02 PDAC cells and treated with Cy/CVAX in combination with IDO1 inhibitor as well as the addition of $100 \mu \mathrm{g}$ anti-PD-L1 antibody intraperitoneally starting on day 5 and continuing twice a week for 3 weeks. (C) Kaplan-Meier survival curves of tumor bearing mice treated with combinations of IDO1 inhibitor in combination with anti-PD-L1 antibody in the absence of CVAX. Data represent results obtained from experiments with 8-10 mice per group that were repeated at least twice. ${ }^{*} P<0.05,{ }^{* *} P<0.01,{ }^{* *} P<0.001$; NS, not significant, by log-rank test.

an immune checkpoint inhibitor such as an IDO1 inhibitor or antiPD-1/PD-L1 blockade antibodies, PDACs need to be primed with a $\mathrm{T}$ cell-inducing agent such as the Cy/GVAX vaccine. Nevertheless, adding anti-PD-L1 antibody on top of the combination of the IDO1 inhibitor and Cy/GVAX led to only a modest, not statistically significant, enhancement of survival (Figure 2B). Moreover, the combination of anti-PD-L1 antibody and IDO1 inhibitor did not result in any improved survival compared with IDO1 inhibitor alone (Figure 2C). Anti-PD-1 antibody showed similar results as anti-PD-L1 antibody (data not shown).

Next, we attempted to understand the mechanism by which IDO1 inhibitor enhanced the antitumor activity of GVAX. To this end, we examined whether IDO1 inhibition can modulate immunosuppressive mechanisms in the tumors. Mice with liver metastases were treated similarly to the above experiment. However, we harvested the livers 2 weeks after tumor implantation, before there was a gross difference in tumor growth among different treatment groups. Tumor infiltrating immune cells were then harvested for flow cytometry analysis. As shown in Figure 3A, similar to our previous observation, $\mathrm{CD} 4^{+} \mathrm{CD} 25^{+} \mathrm{Foxp}^{+}$Tregs in the tumors were found to be still induced by the GVAX treatment in combination with Cy. IDO1 inhibitor was able to suppress Tregs to an intratumoral baseline level but was not able to lower the intratumoral Treg level further. We also used single-stain IHC to examine Foxp3 expression in the PDAC specimens from patients who were treated with GVAX. As shown in Figure 3F, Foxp $3^{+}$cells are significantly higher in the above-described IDO1 ${ }^{\text {hi }}$ PDACs than IDO $1^{\text {lo }}$ PDACs. These results suggest that IDO1 inhibitor may potentiate the antitumor activity of a vaccine therapy by removing the Treg-mediated immune suppressive mechanism induced in the TME to counteract the antitumor activity of the vaccine therapy.

IDO1 is involved in the phenotypic changes and polarization of intratumoral Thelper cells in PDACs. The single-color IHC staining of Foxp3 may not necessarily represent Tregs, so we retrieved the original data from a previously published study that used mul- tiplex IHC staining of multiple $\mathrm{T}$ cell markers to define $\mathrm{T}$ helper cells, including Th0, Th1, Th2, Th17, and Treg, in PDACs from the patients who received the GVAX treatment (26). This cohort of PDACs overlapped with the above cohort of PDACs; thus, IDO1 had already been stained. Among them, 3 PDACs were IDO $1^{\text {hi }}$. As shown in Figure 3, these 3 IDO $^{\text {hi }}$ PDACs have significantly more Th2, Th17, and Tregs than IDO $1^{10}$ PDACs. By contrast, Th1 and Th0 cells were approximately the same in IDO $1^{\text {hi }}$ and in $I D O 1^{\text {lo }}$ PDACs. Consistent with our results IDO $1^{\text {hi }}$ PDACs have significantly more $\mathrm{CD} 8^{+} \mathrm{T}$ cells than IDO1 $1^{\text {lo }}$ PDACs. Although we cannot rule out the possibility that the induction of IDO1 expression is a consequence of high Th2, Th17, and Tregs, the preclinical study (Figure 3A) demonstrates that an IDO1 inhibitor can lower the number of Tregs. Thus, it is more likely that the phenotypic changes of $\mathrm{T}$ helper cells are a consequence of the changes of IDO1 expression. It remains to be determined whether IDO1 plays a direct mechanistic role in the phenotypic changes of $\mathrm{T}$ helper cells and whether the phenotypic changes of $\mathrm{T}$ helper cells represent a change in the $T$ helper cell differentiation or distribution within the tumors.

RNA sequencing was performed on dissected stroma of available slides of PDAC tumor treated with neoadjuvant GVAX prior to pancreatectomy. Tumors were subgrouped by IDO $1^{\text {hi }}$ IHC expression and IDO $1^{1 \mathrm{lo}}$ expression to further investigate those roles in T cell polarization (Figure 4). PDAC tumors with high IDO1 expression were associated with significantly lower IL-2, IL-12A, and IL-12B gene expression, suggesting Th1 differentiation is suppressed by IDO1. Therefore, this may explain why Th1 cells were not significantly increased in the IDO $1^{\text {hi }}$ human PDACs, despite an anticipated increase in the infiltration of Th1 cells driven by vaccine therapy. Conversely, IL-6 gene expression is increased in these IDO $1^{\text {hi }}$ tumors, which may further explain the differentiation skew toward Th17 and Tregs. IL-23 gene expression is also decreased in IDO1 ${ }^{\text {hi }}$ patients. Th17 cells are known to be a highly plastic subset with IL-12/IL-23 helping to push toward a more cytotoxic Th1-like cell that expresses IFN- $\gamma$ and can lose IL-17A and IL-17F expression 

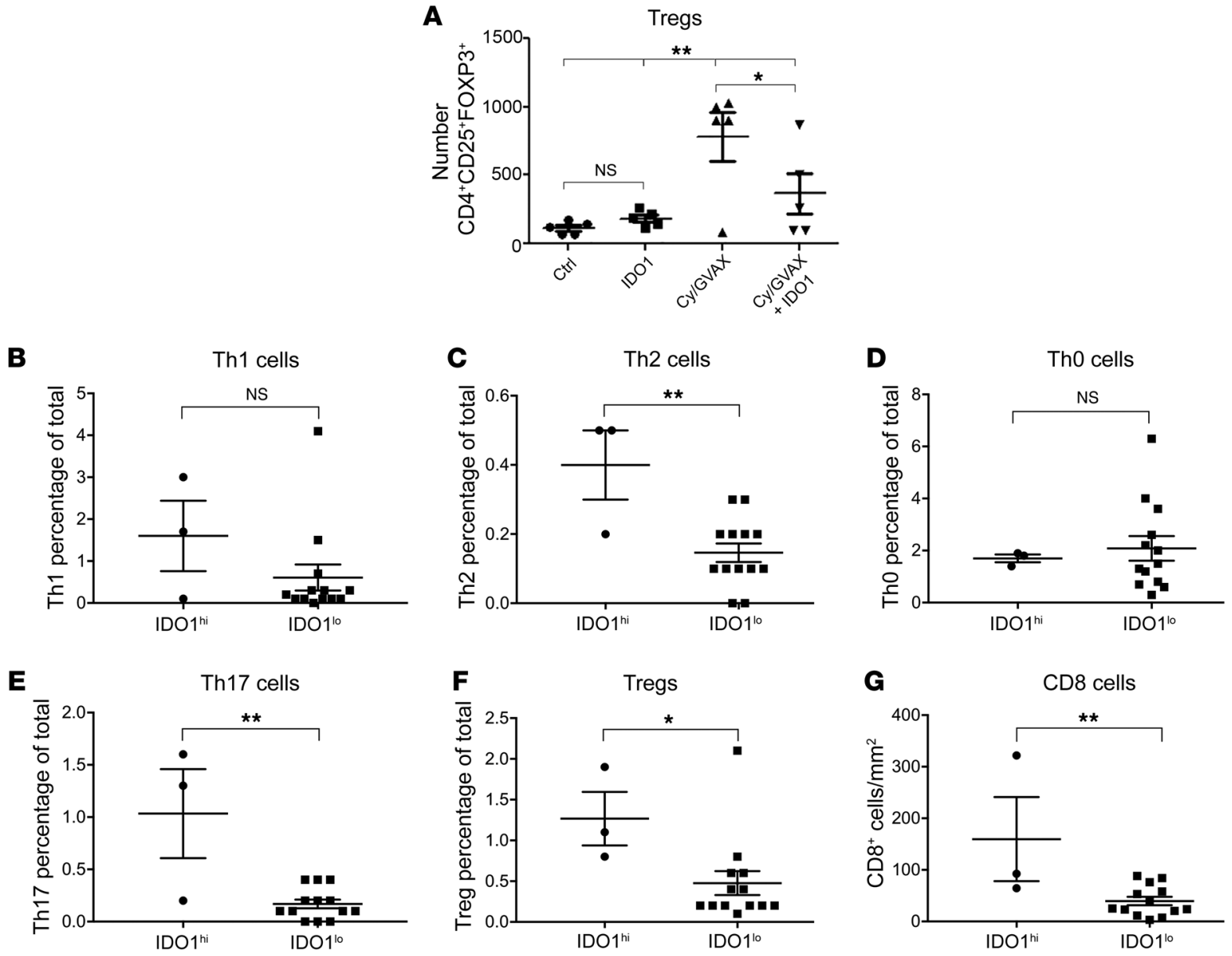

Figure 3. High ID01 expression upregulation is associated with a shift to an antitumor immune cell environment. (A) Total number of Tregs (CD4 ${ }^{+}$CD25 ${ }^{+}$ $\mathrm{Foxp}^{+}$) quantified by flow cytometry analysis in tumor-infiltrating lymphocytes of tumor-bearing mice of the Panc02 hemispleen model and indicated treatments. Data represent mean \pm SEM from one representative experiment of 4-5 mice per treatment group, repeated twice. Comparisons of the (B)

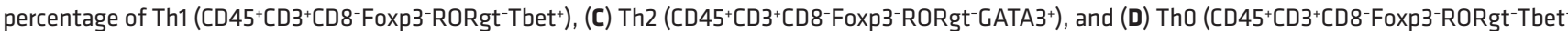

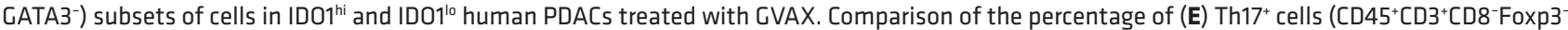

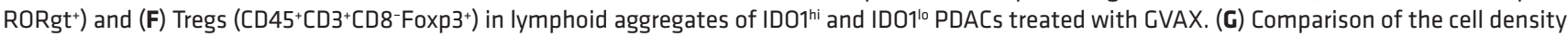
of $\mathrm{CD}^{+}$cells $\left(\mathrm{CD} 45^{+} \mathrm{CD}^{+} \mathrm{CD}^{+}\right)$in IDO1 ${ }^{\text {hi }}$ and ID01/0 PDACs following GVAX treatment $(n=16)$. Expression was quantified using Image Analysis Software (Aperio). ${ }^{*} P<0.05,{ }^{*} P<0.01$; NS, not significant, by unpaired $t$ test.

while maintaining RORgt (27). Our data are consistent with literature that suggests that IL- 6 and TGFb maintain a tumor-promoting Th17 cell subtype $(28,29)$. Moreover, IL-10 is significantly lower in the IDO ${ }^{\text {hi }}$ PDACs than IDO $1^{\text {lo }}$ PDACs that were pretreated with GVAX (Supplemental Figure 2). IFN- $\gamma$ and other type I interferons are known to induce IL-10 expression, and may also induce IL-10 in the GVAX-treated PDACs, concurrently with IDO1. TGFb expression was not significantly changed between $I D O 1^{\text {hi }}$ versus IDO $1^{\text {lo }}$ PDACs, suggesting that other factors, together with IL-6, may play a role in regulating Tregs (Supplemental Figure 2). IL-4 is significantly lower in IDO $1^{\text {hi }}$ PDACs than in IDO1 ${ }^{\text {lo }}$ PDACs, whereas IL-5 is similar between these 2 cohorts of PDACs (Supplemental Figure 2). Therefore, it is not clear whether IDO1 expression plays a direct role in Th2 differentiation. The observed increase in IL-4 expression in IDO1 ${ }^{\text {hi }}$ PDACs could also be a result of $\mathrm{T}$ cell activation by GVAX. Therefore, based on these expression patterns, we conclude that IDO1 expression plays a role in regulating $\mathrm{T}$ helper cell polarization, particularly Th1 and Th17 polarization, and possibly Treg polarization. However, we cannot exclude that Th2 polarization is also affected by IDO1 expression indirectly due to the skew away from Th1 polarization.

As anticipated, we demonstrated a vaccine-induced intratumoral immune cell infiltration. Changes in $\mathrm{T}$ cell trafficking signals were investigated by comparing IDO $1^{\text {hi }}$ versus $I D O 1^{\text {lo }}$ GVAX-treated PDAC tumors. While some significant change, such as CXCL11 expression, was observed, many T cell trafficking signals, including CCL2, CCL5, CXCR3, CXCL9, and CXCL10 (Supplemental Figure 3), were not significantly affected by IDO1 expression (Supplemental Figure 3). Therefore, IDO1 inhibition may not play a major role in regulating $\mathrm{T}$ cell trafficking, or its role is secondary to its role in $\mathrm{T}$ cell polarization (Figure 4).

The role of IDO1 inhibition on T helper cell polarization was subsequently validated in the mouse model of PDAC with isolated $\mathrm{CD}^{+} \mathrm{T}$ cells from treated mice, by assessing gene expression with quantitative real-time reverse transcription polymerase chain reaction (RT-PCR) (Supplemental Figure 4). The results con- 
A
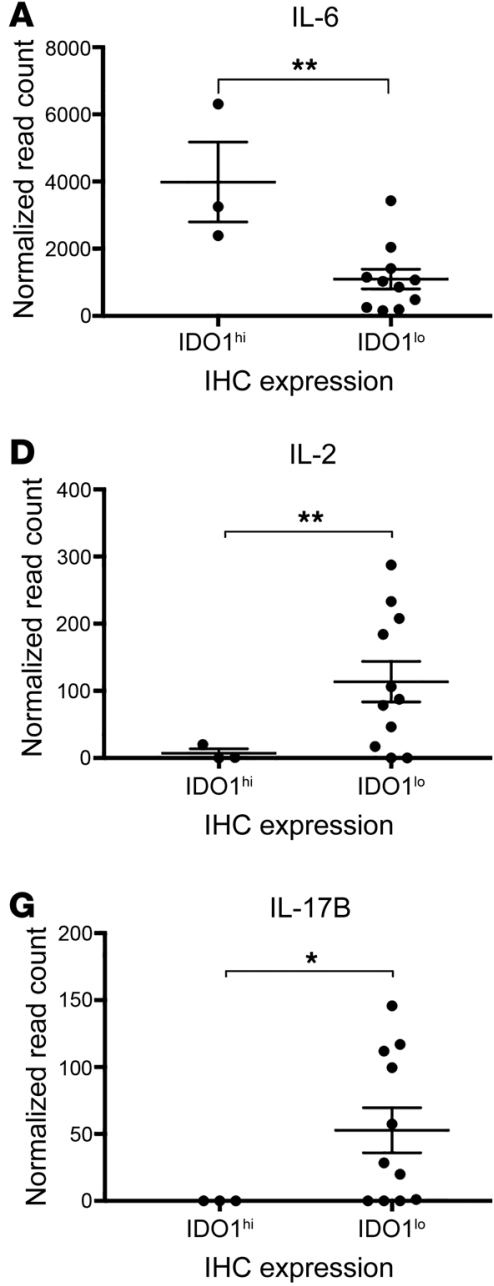

B
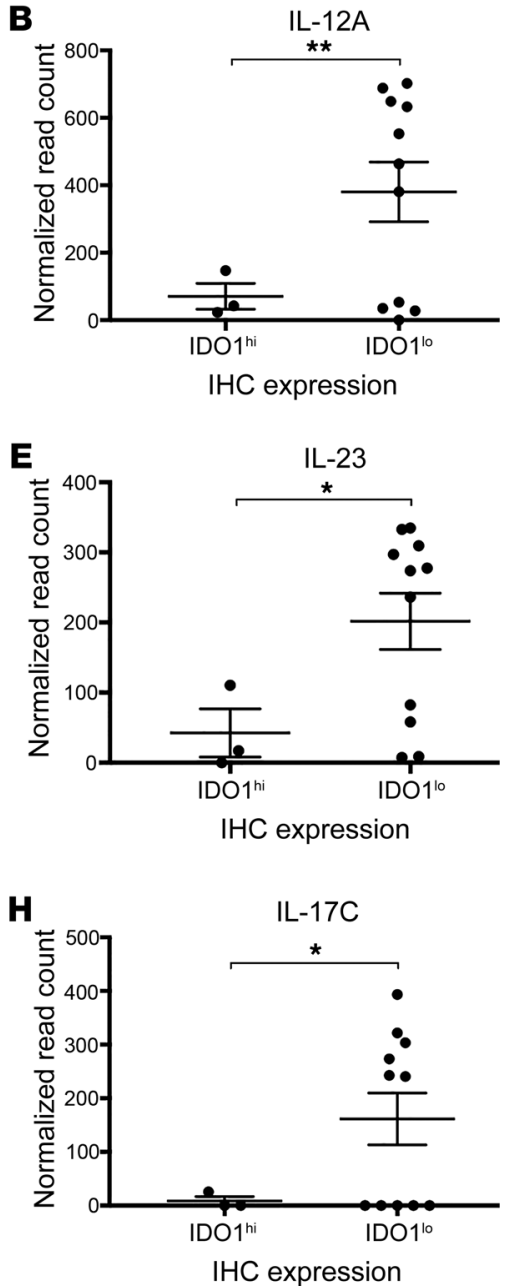

C
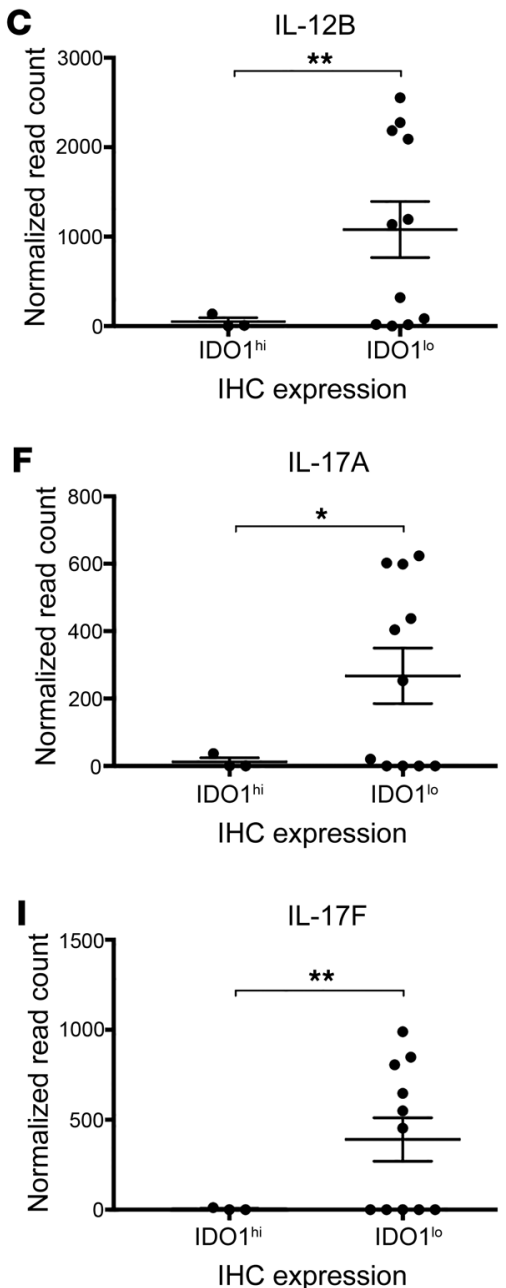

Figure 4. RNA sequencing of dissected stroma of human PDAC tissue. Stroma of representative FFPE tumor sections from patients treated with GVAX were microdissected by a pathologist. RNA was purified and amplified, and RNA sequencing was performed. Read count was normalized to the total prior to analysis. IDO1 $1^{\mathrm{hi}}$ and IDO1 ${ }^{\mathrm{lo}}$ expression was determined by IHC as in Figure 3 for comparative analysis. Expression of IL-6, IL-12A, IL-12B, IL-2, IL-23, IL-17A, IL-17B, IL-17C, and IL-17F was assessed, respectively, as indicated $(\mathbf{A}-\mathbf{I})(n=14) .{ }^{*} P<0.05,{ }^{*} P<0.01$; NS, not significant, by unpaired $t$ test.

firmed that $\mathrm{CD} 4^{+} \mathrm{T}$ cells from tumors treated with IDO1 inhibitor and GVAX have significantly increased IL-2 expression and significantly decreased expression of IL-6 comparing to those treated by GVAX alone (Supplemental Figure 4). As IL-23 and IL-12 are largely expressed by DCs and other myeloid cells, their expression was not assessed in the isolated $\mathrm{CD} 4^{+} \mathrm{T}$ cells. Nevertheless, IL-12 receptor expressed on the $\mathrm{CD}^{+} \mathrm{T}$ cells was not significantly changed in the IDO $1^{\text {hi }}$ tumors, suggesting that the effect is on the signals that regulate the polarization of Th1 cells, not on Th1 cells themselves (Supplemental Figure 2). The expression of IL-17 isoforms was also assessed in isolated $\mathrm{CD} 4^{+} \mathrm{T}$ cells of treated mice. The expression of IL-17A and IL-17F was noted to be decreased or similar following IDO1 inhibitor treatment, whereas expression of IL-17B, C, and D isoforms was increased. Notably, the IFN- $\gamma$ expressing Th1-like Th17 cells can lose the expression of IL-17A and IL-17F which share the same receptor $(27,30,31)$ whereas the less well-studied subunits IL-17B, C, and D may bind to different receptors (32) and appear to be needed in the IL-23/IL-12-induced Th1-like IFN- $\gamma^{+}$Th17 cells, according to our results. Of note, the patterns of IL-17 expression did not directly mirror human data, as all IL-17 isoforms were consistently lower in the IDO ${ }^{\text {hi }}$ human PDAC tumors compared with IDO $1^{\text {lo }}$ human PDAC tumors (Figure 4) (Supplemental Figure 2). It should be noted that IL-17 expression in human PDAC tumors was examined in dissected stroma, which includes cell types beyond $\mathrm{CD}^{+} \mathrm{T}$ cells that may also contribute to IL-17A/F expression. When IDO1 is inhibited, intratumor Tregs are significantly reduced in the mouse PDAC model (Figure 3A), suggesting that IDO1 likely plays a role in regulating Tregs. Consistent with human data (Supplemental Figure 2), IL-10 was not significantly affected by IDO1 inhibitor treatment in the mouse PDAC model (Supplemental Figure 4). In addition, IL-4 and $\mathrm{TGFb}$ in $\mathrm{CD}^{+} \mathrm{T}$ cells were not significantly affected by IDO1 inhibitor treatment (Supplemental Figure 4). Therefore, our study further supports the hypothesis that IDO1 inhibition plays a role in regulating $\mathrm{T}$ helper cell polarization, particularly Th1 and Th17 polarization, and possibly Treg polarization.

These results suggest that it would be important to inhibit IDO1 in the setting of vaccine therapy; otherwise, the TME would skew toward Th2 and Tregs in those IDO $1^{\text {hi }}$ PDACs. Ironically, the IDO $1^{\text {hi }}$ PDACs were also infiltrated with higher numbers of 
A $\% \mathrm{CD}^{+}$out of $\mathrm{CD}^{+}$population

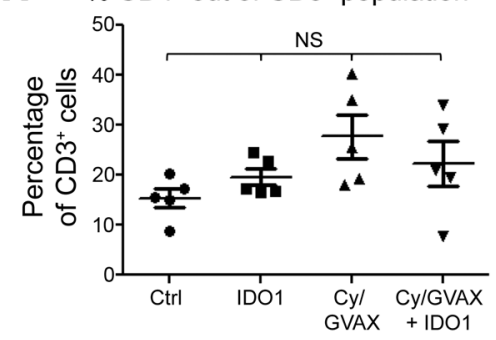

B $\% \mathrm{CD}^{+}$out of $\mathrm{CD}^{+}$population

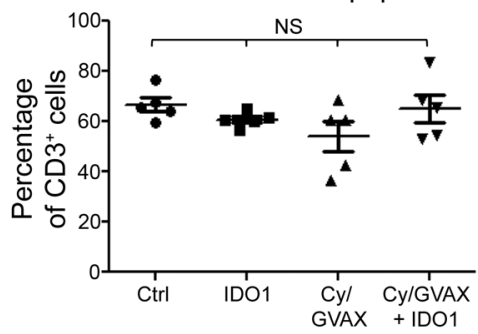

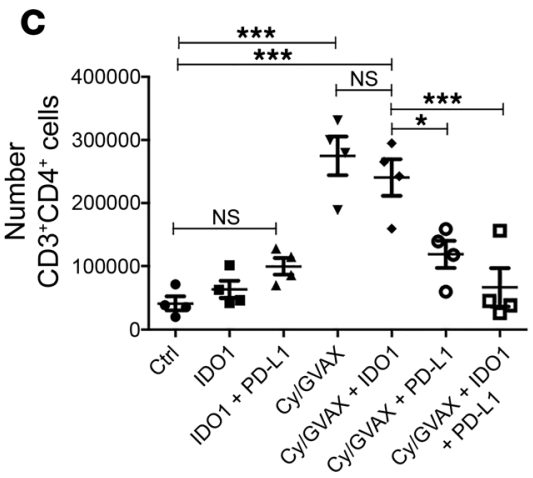

D

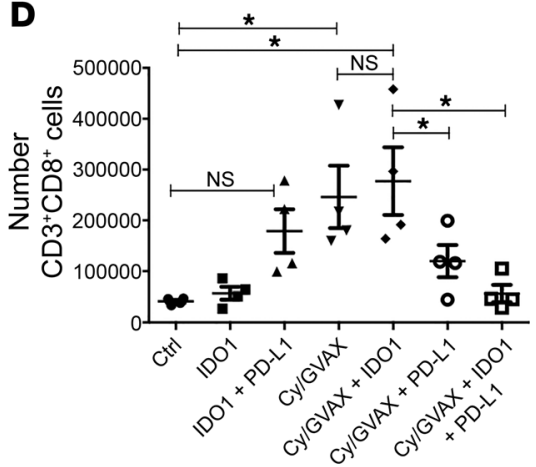

$\mathbf{E}$
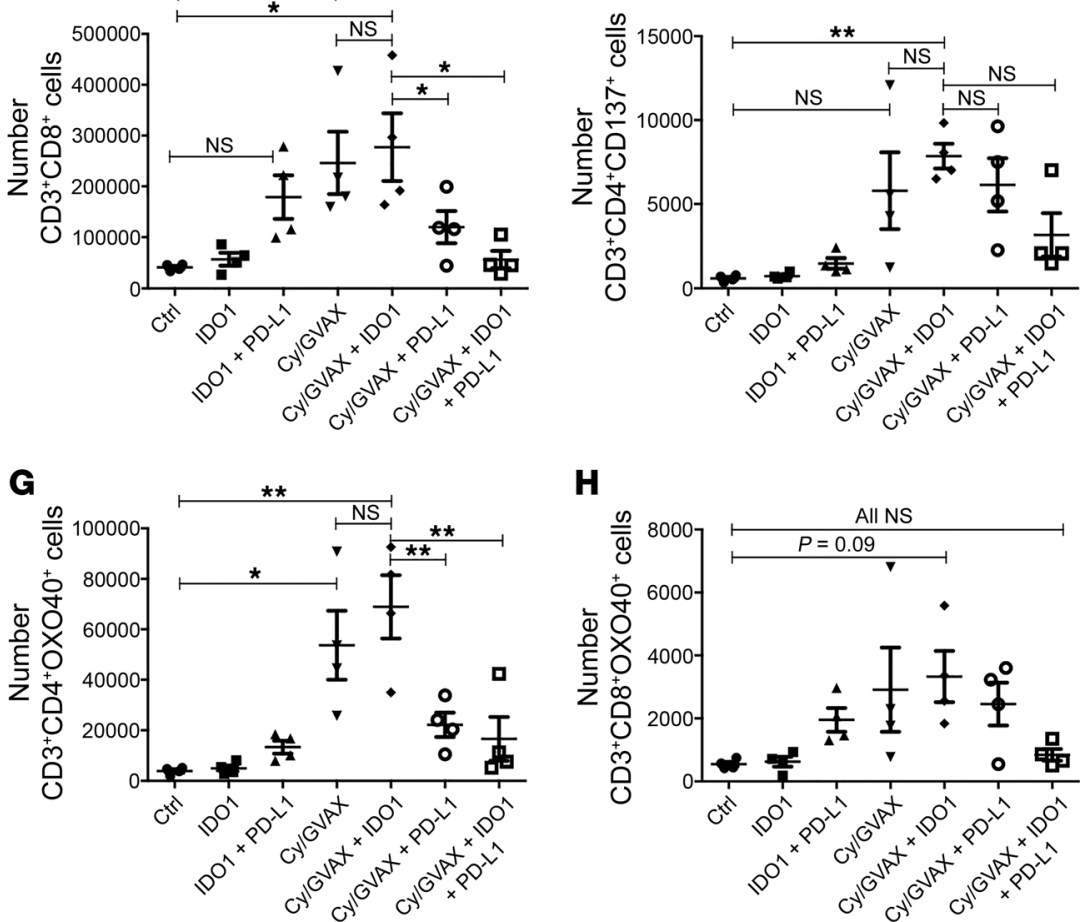

H

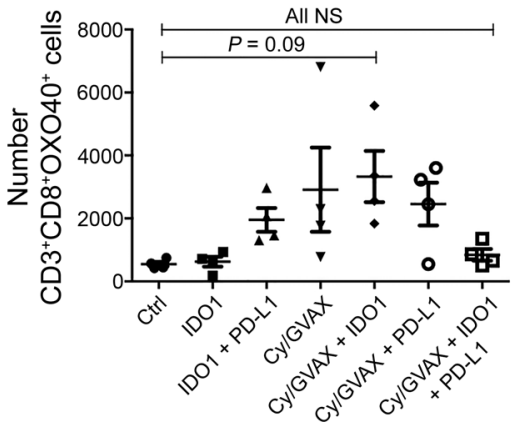

Figure 5. ID01 inhibitor in combination with GVAX increases effector T cell infiltration without added benefit from additional anti-PD-L1 antibody. Percentages of (A) $\mathrm{CD} 4^{+}$cells and (B) $\mathrm{CD} 8^{+}$cells out of the $\mathrm{CD}^{+}$population as quantified by flow cytometry analysis in tumor-infiltrating lymphocytes isolated from

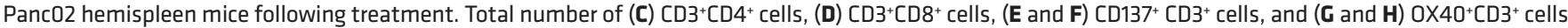
as quantified by flow cytometry analysis from the same set of Panc02 hemispleen mice following treatment. Data represent mean \pm SEM from one representative experiment of 4-5 mice per treatment group, repeated twice. ${ }^{*} P<0.05$, ${ }^{* *} P<0.01$, ${ }^{* * *} P<0.001$; NS, not significant, by 1-way ANOVA.

$\mathrm{CD}^{+} \mathrm{T}$ cells and, as shown previously in Figure 3G, individuals with PDACs with higher numbers of $\mathrm{CD}^{+} \mathrm{T}$ cells are most likely to benefit from the vaccine therapy. This further underlines the potential critical importance of IDO1 inhibition in patients who may be treated with a vaccine therapy.

The combination of Cy/GVAX and IDO1 inhibitor enhanced intratumoral effector $T$ cell infiltration and function, but adding antiPD-L1 antibody to the combination of Cy/GVAX and IDO1 inhibitor decreased effector $T$ cell infiltration and function. Our results suggest that the primary mechanistic role of IDO1 may alter the T cell populations within the tumor. As the human data analysis suggested that IDO1 ${ }^{\text {hi }}$ PDACs were also infiltrated with higher numbers of $\mathrm{CD}^{+} \mathrm{T}$ cells following vaccine therapy, one concern would be whether IDO1 inhibitor would lower the number of $\mathrm{CD}^{+} \mathrm{T}$ cells. As shown in Figure 5, A and B, the percentage of $\mathrm{CD}^{+}$or $\mathrm{CD}^{+}$ cells in CD3 cells were not significantly changed among all treatment groups. Nevertheless, the absolute numbers of $\mathrm{CD}^{+}$and
$\mathrm{CD} 8^{+} \mathrm{T}$ cells in the tumors increased significantly (Figure $5, \mathrm{C}$ and D) in the group treated with $\mathrm{Cy} / \mathrm{GVAX}$ or the combination of $\mathrm{Cy} /$ GVAX and IDO1 inhibitor compared with the groups left untreated or treated with IDO1 inhibitor alone. There is no significant difference between Cy/GVAX and the combination of Cy/GVAX and IDO1 inhibitor, suggesting that IDO1 inhibitor does not inhibit $\mathrm{CD}^{+} \mathrm{T}$ cells. However, the addition of anti-PD-L1 antibody to the combination of Cy/GVAX and IDO1 inhibitor significantly lowered tumor-infiltrating $\mathrm{CD}^{+}$and $\mathrm{CD} 8^{+} \mathrm{T}$ cells to a level similar to that in the untreated tumors (Figure 5, C and D). Intratumoral T cells that expressed activation markers such as OX40 and CD137 were also significantly (Figure 5, E-H) increased in the Cy/GVAX-treated group and further increased with a trend in the group treated with the combination of Cy/GVAX and IDO1 inhibitor. Although adding anti-PD-L1 antibody to Cy/GVAX was able to maintain the levels of $\mathrm{OX} 40^{+}$or $\mathrm{CD} 137^{+} \mathrm{T}$ cells at a level similar to that in the mice treated by Cy/GVAX alone, adding anti-PD-L1 antibody to the 
A

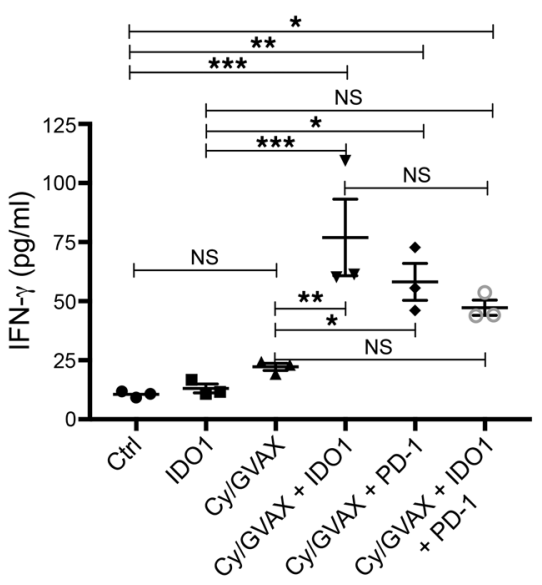

Treatment
B

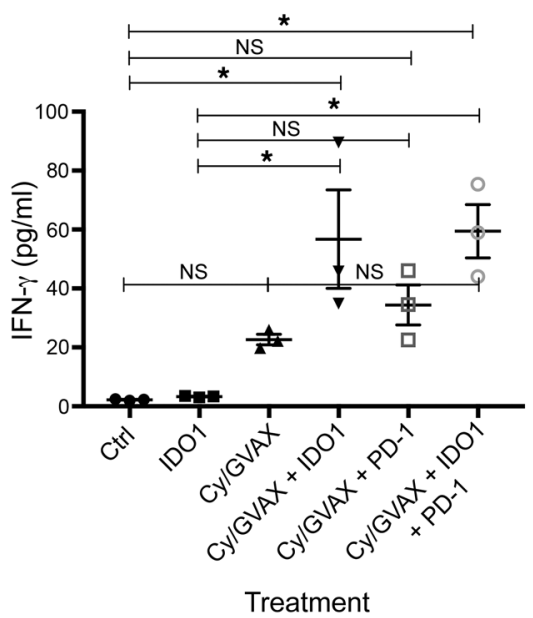

Figure 6. ID01 inhibitor in combination with GVAX increases IFN- $\gamma$ expression compared with singleagent therapies. $\mathrm{CD} 8^{+} \mathrm{T}$ cells were isolated from purified (A) livers and (B) spleen on day 14 following hemispleen injection of $2 \times 10^{6}$ Panc 02 tumor cells. Mice were treated with ID01 inhibitor, Cy, GVAX, and anti-PD- 1 antibody as listed. IgC and methocel controls were used. Irradiated Panc02 tumor cells were used as antigenic targets for isolated CD8 ${ }^{+}$cells for ELISA analysis. Each experimental group consisted of 5 mice pooled and analyzed in triplicate. Data represent mean \pm SEM from one representative experiment that was repeated. ${ }^{*} P<$ $0.05,{ }^{* *} P<0.01,{ }^{* * *} P<0.001$; NS, not significant, by 1-way ANOVA. combination of Cy/GVAX and IDO1 inhibitor significantly lowered $\mathrm{OX} 40^{+}$or $\mathrm{CD} 137^{+} \mathrm{T}$ cells (Figure 5, E-H). It should be noted that intratumoral $\mathrm{OX} 40^{+} \mathrm{CD} 4^{+} \mathrm{T}$ cells in the tumors treated with the combination of Cy/GVAX and IDO1 inhibitor were significantly higher than those treated with the combination of Cy/GVAX and anti-PD-L1 antibody. In order to investigate the mechanism of this decrease in T cell infiltration, flow cytometry analysis of TILs was performed and revealed that tumors treated with combination GVAX, IDO1 inhibitor, and anti-PD-L1 antibody had a statistically significant increase in the Annexin $\mathrm{V}^{+}$, apoptotic $\mathrm{CD}^{+} \mathrm{CD} 8^{+}$cells as compared with other treatment groups (Supplemental Figure 5). RNA was extracted from $\mathrm{CD}^{+} \mathrm{T}$ cells isolated from treated tumors to assess changes in T cell trafficking chemokine receptors such as CXCR3 by RT-PCR. A modest trend without statistical significance was noted with a decrease in $\mathrm{Cxcr} 3$ gene expression in mice receiving the combination of Cy/GVAX, IDO1 inhibitor, and anti-PD-L1 antibody compared with mice receiving the combination of Cy/GVAX and IDO1 inhibitor or Cy/GVAX alone. The effect of IDO1 inhibition on cytokines/cytokine receptors cannot be ruled out; however, it does not appear to play a major role in regulating the $\mathrm{CD}^{+} \mathrm{T}$ cell trafficking (Supplemental Figure 5). $\mathrm{CD}^{+}$IFN- $\gamma$ expression was increased with the addition of IDO1 inhibitor to Cy/GVAX compared with Cy/GVAX alone (Supplemental Figure 5). Eomes gene expression was decreased in isolated $\mathrm{CD}^{+}$cells in mice receiving the combination of Cy/GVAX, IDO1 inhibitor, and anti-PD-L1 antibody compared with the combination of Cy/GVAX and IDO1 inhibitor or Cy/GVAX alone. Taken together, these results suggest that although there are fewer infiltrating $\mathrm{CD}^{+}$cells in the tumors treated by the combination of $\mathrm{Cy} /$ GVAX, IDO1 inhibitor, and anti-PD-L1 antibody, these CD8 ${ }^{+}$cells may be less exhausted (Supplemental Figure 5) than $\mathrm{CD}^{+}$cells in tumors treated another way.

Consistent with prior studies, we showed that $\mathrm{CD}^{+} \mathrm{T}$ cells isolated from tumor-infiltrating immune cells of tumor-bearing mice that were treated with Cy/GVAX demonstrated effector cell function as reflected by IFN- $\gamma$ expression in response to the stimulation of irradiated Panc02 tumor cells. However, a significantly enhanced tumor-specific effector $\mathrm{T}$ cell function was demonstrated in $\mathrm{CD}^{+} \mathrm{T}$ cells isolated from tumor infiltrating immune cells from mice that were treated with the combination of Cy/GVAX and IDO1 inhibitor compared with those that were treated by Cy/GVAX alone (Figure 6A and Supplemental Figure 5D). This result suggests that the tumor-specific effector T cell function is enhanced by $\mathrm{Cy} /$ GVAX and further enhanced by adding the IDO1 inhibitor to $\mathrm{Cy} /$ GVAX. It should be noted that the combination of Cy/GVAX and IDO1 inhibitor led to a trend toward stronger antitumor effector $\mathrm{T}$ cell function than the combination of Cy/GVAX and anti-PD-1 antibody. However, adding anti-PD-L1 antibody to the combination of Cy/GVAX and IDO1 inhibitor enhanced this effector T cell function in the peripheral lymphocytes (Figure 6B), but did not further enhance this effector $\mathrm{T}$ cell function in the tumors; instead, a trend toward decreased intratumoral effector $\mathrm{T}$ cell function was observed. Similarly, adding anti-PD-1 antibody to the combination of Cy/GVAX and IDO1 inhibitor had a similar effect as adding antiPD-L1 antibody. These results provide a mechanistic explanation of why the combination of IDO1 inhibitor and anti-PD-L1 antibody does not yield an additional antitumor effect on top of IDO1 inhibitor or anti-PD-L1/PD-1 antibody, respectively.

IDO1 inhibitor does not modulate intratumoral myeloid-derived suppressor cells quantitatively, but qualitatively. IDO1 inhibitor is also thought to be a modulator of MDSCs. Therefore, we compared the intratumoral myeloid cell infiltrations between IDO1 ${ }^{\text {hi }}$ and IDO $^{\text {lo }}$ human PDACs, but did not notice any significant difference in all types of myeloid lineage cells that were defined in the previous study (Supplemental Figure 6). Nevertheless, MDSCs are still not well defined in human tissues. Therefore, we analyzed MDSCs, as defined by the cell surface expression patterns of CD11b, Ly6C, Ly6G, and F4/80, in the tumors from mice treated as described earlier. As shown in Figure 7A, the total number of myeloid cells in mice was significantly induced by the $\mathrm{Cy} /$ GVAX treatment, whereas IDO1 inhibitor alone does not change the myeloid cell populations. Adding IDO1 inhibitor to the $\mathrm{Cy} /$ GVAX treatment, however, lowered the total number of intratumoral myeloid cells significantly, but did not change the intratumoral percentage of myeloid cells among all the cells in the liver metastases (Figure 7B). Statistically nonsignificant trends were noted in repeated experiments with variability likely related to the difference in tumor size of corresponding treatment groups (Sup- 
A

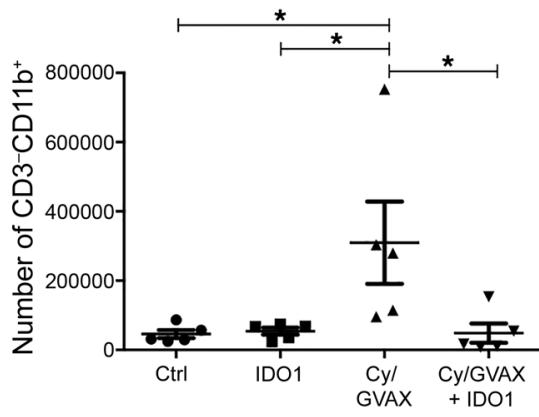

D

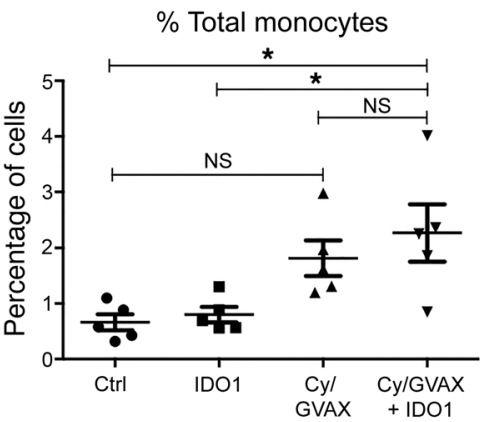

G

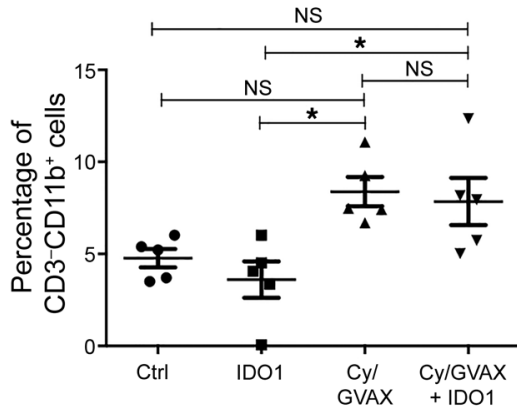

B

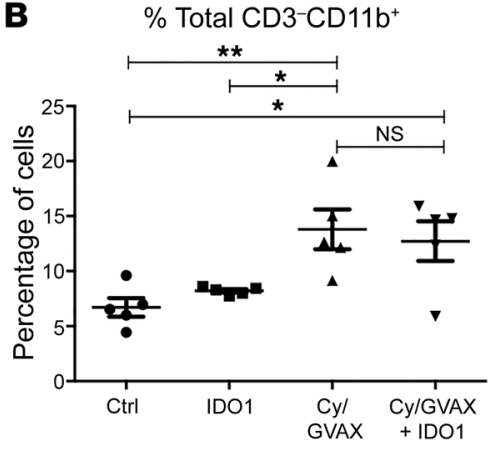

E

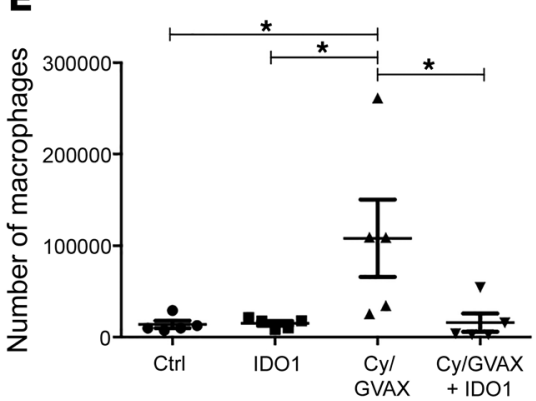

H $\%$ G-MDSC out of CD3-CD11 $\mathrm{b}^{+}$

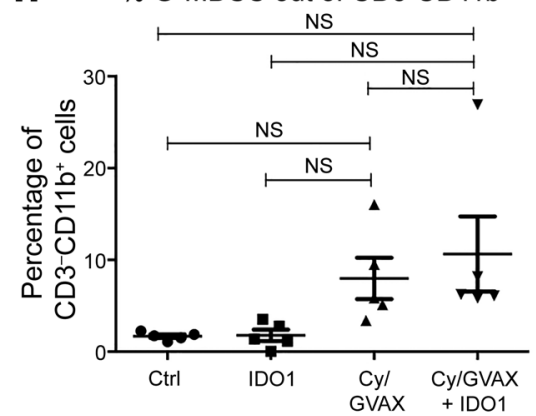

C

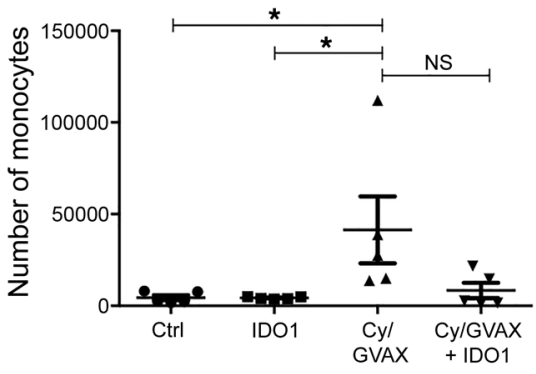

$\mathbf{F}$
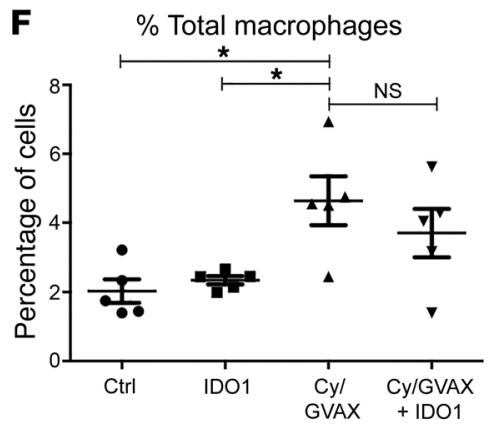

I

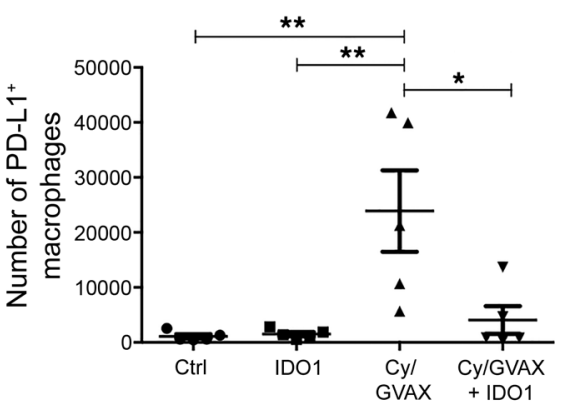

Figure 7. IDO1 inhibitor in combination with GVAX lowered the total number of macrophages and monocytes compared with GVAX treatment alone, although it did not change the percentage of myeloid-derived suppressor cells. Mice underwent hemispleen procedure receiving $2 \times 10^{6} \mathrm{Panc} 02 \mathrm{PDAC}$ cells followed by administration of $100 \mathrm{mg} / \mathrm{kg}$ Cy on day 3 and GVAX on day 4 . ID01 inhibitor $(200 \mu \mathrm{g} / \mathrm{kg})$ was administered by oral gavage twice a day starting on day 3. Mice were sacrificed at day 13 for flow cytometry analysis. (A) Total numbers and (B) percentage of myeloid cells (CD3-CD11b+) on flow cytometry analysis within the total tumor-infiltrating lymphocytes (TILs) of Panc02 hemispleen mice following treatment. (C) Total number and (D) per-

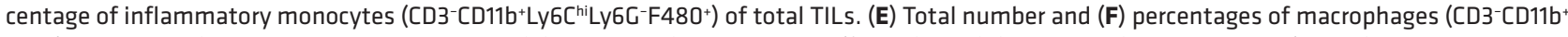

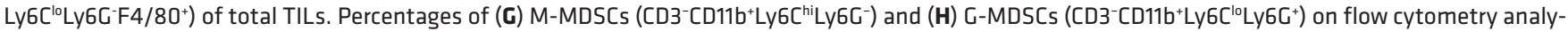

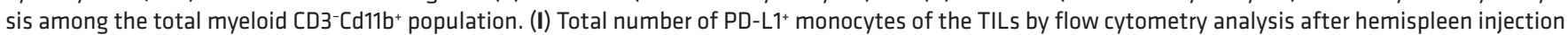
of Panc02 cells and indicated therapy. Data represent mean \pm SEM from one representative experiment of 4-5 mice per treatment group, repeated twice. ${ }^{*} P<0.05,{ }^{* *} P<0.01$; NS, not significant, by 1-way ANOVA.

plemental Figure 7). The decrease in the number of total myeloid cells in mice treated with the combination of Cy/GVAX and IDO1 inhibitor is thus likely due to the decrease in the overall cell number of the liver metastases and thus an antitumor treatment effect. Within the total myeloid cell population, IDO1 consistently lowered the number of monocytes and macrophages, but did not lower the percentage of macrophages among all the cells in the liver metastases (Figure 7, C-F). This is consistent with our human data. The percentages of granulocytic MDSCs (G-MDSCs) and monocytic MDSCs (M-MDSCs) among the myeloid cells were also increased by the Cy/GVAX treatment, suggesting that the increase of MDSCs is not merely the consequence of the increase of the overall myeloid cell population (Figure 7, G and H). Notably however, IDO1 inhibitor did not change the percentage of G-MDSCs and M-MDSCs among myeloid cells (Figure 7). We further examined the number of PD-L1 ${ }^{+}$macrophages or monocytes in the liver metastases. As shown in Figure 7I, PD- $1^{+}$macrophages were induced by $\mathrm{Cy} / \mathrm{GVAX}$ treatment, but were significantly decreased by IDO1 inhibitor, suggesting that IDO1 inhibitor is able to play the role of anti-PD-1/PD-L1 antibodies by suppressing the PD-L1 signaling in macrophages. Because PD-L1 ${ }^{+}$MDSCs or PD-L1 ${ }^{+}$monocytes were exceptionally uncommon in all treatment groups, we were not able to adequately assess the effects of IDO1 inhibitor on these myeloid cell subsets (Supplemental Figure 8). These results 
A

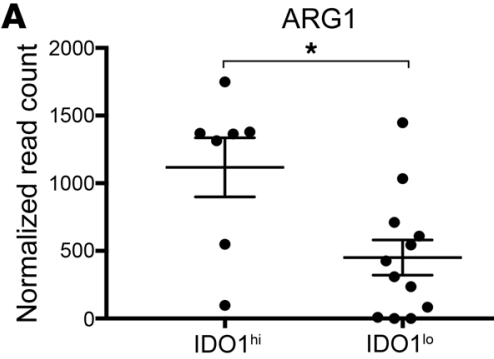

Stromal IDO1 expression

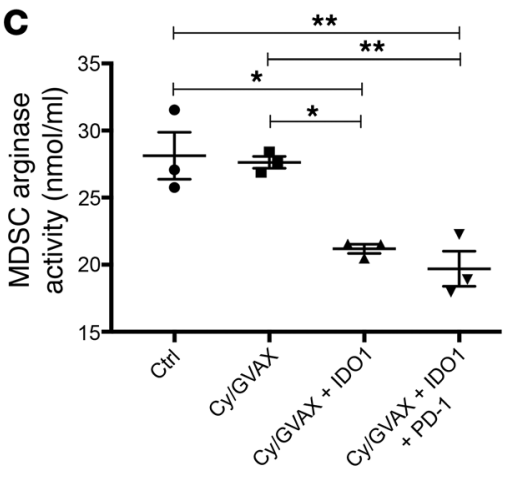

B

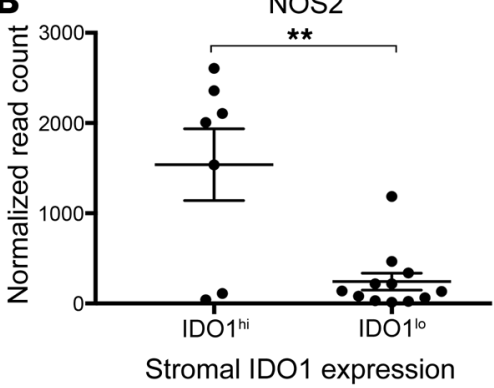

D

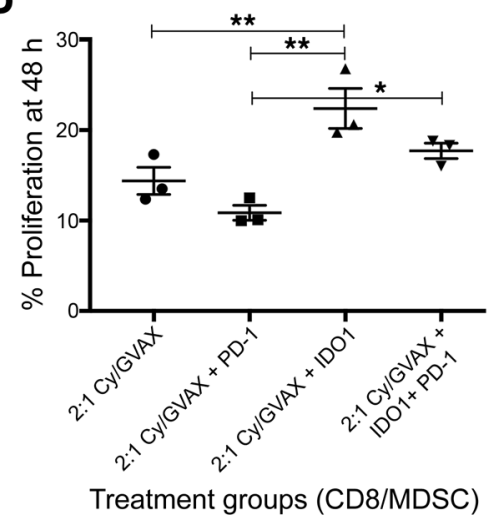

Figure 8. T cell suppression function of MDSCs was diminished by IDO1 inhibitor through reducing production of the MDSC suppressive factors. Stroma of representative FFPE tumor sections from patients treated with GVAX were microdissected by a pathologist. RNA was purified and amplified, and RNA sequencing was performed. Read count was normalized to the total prior to analysis. IDO $1^{\text {hi }}$ and $\mathrm{IDO} 1^{10}$ expression was determined by normalized stromal ID01 expression for comparative analysis of (A) ARG1 and $(B)$ NOS2 gene expression $(n=19)$. Mice underwent hemispleen procedure receiving $2 \times 10^{6}$ Panc 02 PDAC cells followed by administration of $100 \mathrm{mg} / \mathrm{kg}$ Cy on day 3 and GVAX on day 4. IDO1 inhibitor $(200 \mu \mathrm{g} / \mathrm{kg})$ was administered by oral gavage twice a day starting on day 3 and continuing for 13 days. Anti-PD-1 antibody $(100 \mu \mathrm{g})$ or IgG control $(100 \mu \mathrm{g})$ was given intraperitoneally starting on day 5 and continuing twice a week. At day 13 , mice were sacrificed and MDSCs were isolated and used for (C) colorimetric arginase assay or (D) T cell suppression assay via coculture experiment in which MDSCs were cocultured for 48 hours with wild-type $\mathrm{CD} 8^{+}$cells stained with CFSE. Flow cytometry diffusion analysis was performed to assess suppressive ability of treated MDSCs. The percentage of $C D 8^{+} T$ cells that divided at least once were calculated based on fluorescence diffusion. Data represent mean \pm SEM of one representative experiment of 5 mice per treatment group pooled and analyzed in triplicate, repeated once. ${ }^{*} P<0.05,{ }^{* *} P<0.01$, by unpaired $t$ test and 1-way ANOVA. suggest that IDO1 inhibitor may decrease the total number of myeloid cells induced by Cy/GVAX. However, despite changes in inflammatory monocytes and tumor-associated macrophages, IDO1 inhibitor does not substantially modulate intratumoral MDSCs quantitatively.

We then assessed the function of intratumoral MDSCs in suppressing the proliferation of $\mathrm{CD}^{+} \mathrm{T}$ cells. To study the effects of IDO1 expression in MDSCs, RNA sequencing of human PDAC tissue was utilized to assess the expression of genes that mediate MDSC suppressive activity, thereby confirming that these factors are downregulated in IDO ${ }^{\text {lo }}$ expressing subset and indirectly suggesting the effect of IDO1 inhibition on MDSC could be mediated by the same mechanism. In the GVAX-treated human PDAC tumors, there was a correlation between stromal IDO1 expression and factors such as $A R G 1$ and NOS2, which are associated with the suppressive function of MDSCs (Figure 8, A and B). Nevertheless, it remains to be tested whether the stromal expression of $A R G 1$ and NOS2 is attributed to myeloid cells.

Mouse PDAC tumors treated with IDO1 inhibitor were utilized to further investigate the effects of IDO1 inhibition on MDSC function. Cy/GVAX treatment itself had the effect of substantially lowering nitrate production compared with untreated controls (data not shown), thus this study focused on assessing arginase activity of MDSCs isolated from treated mice by colorimetric arginase assay. Mouse PDACs treated with IDO1 inhibitor were found to have significantly decreased MDSC arginase activity compared with those left untreated or those treated with Cy/GVAX alone (Figure 8C). Therefore, although the total number of intratumoral MDSCs was not reduced, likely secondary to an effect of the vaccine treatment, the $\mathrm{T}$ cell suppression function of MDSCs was diminished by IDO1 inhibitor, likely through reducing the production of the suppressive factors. These results are consistent with the $\mathrm{T}$ cell suppression assay. In this assay, we found that MDSCs from the tumors of mice treated with Cy/GVAX alone were able to suppress $\mathrm{CD} 8^{+} \mathrm{T}$ cell proliferation. However, MDSCs from the tumors of mice treated with the combination of Cy/GVAX and IDO1 inhibitor showed significantly less suppressive activity on T cell proliferation (Figure 8D). By contrast, although MDSCs from the tumors of mice treated with the combination of Cy/GVAX, IDO1 inhibitor, and anti-PD-1 antibody showed significantly less suppressive activity compared with those from the tumors of mice treated with the combination of $\mathrm{Cy} /$ GVAX, a trend showed MDSCs had more suppressive activity compared with those from the tumors of mice treated with the combination of Cy/GVAX and IDO1 inhibitor. Moreover, MDSCs from the tumors of mice treated with the combination of Cy/GVAX and anti-PD-1 antibody had essentially the same suppressive activity as those from the tumors of mice treated with the Cy/GVAX alone. These results may offer an explanation for why the combination of IDO1 inhibitor and anti-PD-1 antibody does not yield an additional antitumor effect on top of IDO1 inhibitor.

\section{Discussion}

We believe that this study was the first of its kind to test the efficacy of the combination of a vaccine therapy and an IDO1 inhibitor in a murine tumor model. The results supported the development of combination immunotherapy with both cancer vaccine and IDO1 inhibitor. The mechanism of action appears to be similar to our observation regarding the combination of cancer vaccine and anti-PD-1 antibodies, suggesting that vaccine therapy primes the tumor microenvironment of PDACs for both IDO1 inhibitor and anti-PD-1 antibody therapy. IDO1 expression appears to be induced by effector $\mathrm{T}$ cell infiltration and inflammatory cytokines 
as a result of vaccine therapy. The induction of IDO1 expression further influences the type of $\mathrm{T}$ helper cells that dominate the tumor microenvironment. More specifically, Th0 cells were not affected by IDO1 expression, but the tumor microenvironment in IDO1 ${ }^{\text {hi }}$ PDACs is skewed toward Th2, Th17, and Treg cells.

Our study also supports the hypothesis that IDO1 inhibition plays a role in regulating $\mathrm{T}$ helper cell polarization, particularly Th1 and Th17 polarization, and possibly Treg polarization. Although vaccine alone had only a moderate antitumor effect for PDACs, when combined with the IDO1 inhibitor treatment, vaccine demonstrated a significantly improved antitumor efficacy.

The mechanisms of action of the combination of IDO1 inhibitor and vaccine appear to be as follows. First, IDO1 inhibitor targets IDO1-expressing tumor cells, resulting in a downstream effect on the regulation of $\mathrm{CD}^{+}$and $\mathrm{CD} 4^{+}$cells. Specifically, IDO1 plays a role in $\mathrm{T}$ helper cell polarization, particularly in the Th1 and Th17 cells, and possibly in Tregs. Second, IDO1 inhibitor lowers both vaccine-induced intratumoral Tregs and PD-L1-expressing tumor-associated macrophages, although the number of MDSCs does not appear to be affected. The $\mathrm{T}$ cell suppression function of MDSCs was diminished by IDO1 inhibitor through reducing production of the MDSC suppressive factors. IDO1 inhibitor further enhanced vaccine-induced effector $\mathrm{T}$ cell infiltration and tumor-specific cytotoxic $\mathrm{T}$ cell activity. The infiltration of activated $\mathrm{T}$ cells was also enhanced by the IDO1 inhibitor. Despite this synergistic effect on vaccine therapy, IDO1 inhibitor itself lacked these abilities, explaining why the single agent IDO1 inhibitor did not have antitumor efficacy in PDACs. The combination of vaccine and IDO1 inhibitor appeared to have a stronger effect in driving the intratumoral effector $\mathrm{T}$ cell infiltration than the combination of vaccine and anti-PD-L1 antibody. More strikingly, addition of anti-PD-L1 antibody to the combination of IDO1 inhibitor and vaccine substantially lowered the number of intratumoral effector $\mathrm{T}$ cells and activated $\mathrm{T}$ cells to a level almost equivalent to untreated tumors. Nevertheless, in most of our experiments, we found that the combination of vaccine and anti-PD-1/PD-L1 antibody has a similar antitumor efficacy as the combination of vaccine and IDO1 inhibitor. However, the combination of vaccine and IDO1 inhibitor may very likely be more effective in treating human PDACs, which is more heterogeneous than the mouse models of PDACs. A stronger immune response induced by IDO1 inhibitor may result in higher or more durable tumor responses in human PDACs.

In the presence of vaccine therapy, IDO1 inhibitor did not appear to have major activity in modulating myeloid cells or MDSCs quantitatively. Despite this, we found that in tumors treated with the combination of vaccine and IDO1 inhibitor, MDSCs became markedly less suppressive in regard to arginase activity and inhibiting $\mathrm{T}$ cell proliferation. These results suggest that while the major target of IDO1 inhibitor is likely the IDO1-expressing tumor cells with downstream effects on regulation of $\mathrm{CD}^{+}$and $\mathrm{CD}^{+} \mathrm{T}$ cells, the stromal cells, including the myeloid subtype, may also be targeted. IDO1 inhibitor may modulate the MDSC's $\mathrm{T}$ cell suppression function or may affect the interaction between MDSCs and T cells.

One of the most important findings in this study is that there is no synergy in antitumor efficacy between IDO1 inhibitor and anti-PD-1/PD-L1 antibody in combination with vaccine therapy.
This is consistent with the results of the phase III clinical trial of IDO1 inhibitor and pembrolizumab in melanoma (21). The results of this preclinical study suggested that the mechanisms of action of IDO1 inhibitor are centered on T cells, thus potentially only $\mathrm{T}$ cell-inflamed melanoma would have responded to the IDO1 inhibitor treatment. This subgroup of melanomas is also sensitive to immune checkpoint inhibitors. This may explain why the combination of IDO1 inhibitor and anti-PD-1 antibody does not demonstrate an improved survival in melanoma. Although PDACs are immunologically cold tumors, vaccine therapy can convert them into $\mathrm{T}$ cell inflamed hot tumors as shown previously (5). Although IDO1 inhibition or PD-1 blockade is synergized with vaccine treatment for PDAC, the combination of both inhibitor agents with vaccine treatment may have impaired vaccine-induced early $\mathrm{T}$ cell activation. Similarly, a previous publication showed that concomitant treatment with anti-PD-1 antagonist at the time of initiation of anti-OX40 agonist treatment with vaccine resulted in apoptosis of $\mathrm{CD}^{+} \mathrm{T}$ cells (33). Thus, our findings also suggest that initial PD-1 signaling may be required for the effect of the combination treatment of vaccine and IDO1 inhibitor during early $\mathrm{T}$ cell activation and proliferation and that PD-1 pathway blockade in the setting of the combination of vaccine and IDO1 inhibitor may lead to apoptosis of $\mathrm{CD}^{+} \mathrm{T}$ cells. Thus, this preclinical study of T cell-inflamed PDACs may serve as an analog to the situation in melanoma, providing an explanation to the potential redundant nature and lack of synergy of combination anti-PD-1/PD-L1 antibody and IDO1 inhibitor. Further dissection of the underlying mechanisms is warranted in future studies.

We did consistently observe that adding anti-PD-1 or antiPD-L1 antibody to Cy/GVAX and IDO1 inhibitor slightly improved survival in the murine model of PDACs. This may be explained by the effect of IDO1 inhibitor on Tregs and MDSCs. This effect did not yield a significant improvement in survival, likely due to the negative impact on the effector T cells when combining antiPD-1/PD-L1 antibody with IDO1 inhibitor in the presence of vaccine therapy. It is possible that this negative impact of combining anti-PD-1/PD-L1 antibody with IDO1 inhibitor on the effector T cells counteracts the effect of IDO1 inhibitor on Tregs and MDSCs. The underlying mechanism remains to be further explored.

Our study supports the combination of IDO1 inhibitor and vaccine therapy for PDAC treatment. However, our results are limited by the differences between the mouse model of PDAC and human pancreatic cancer. Therefore, further testing in clinical trials of PDACs is warranted. Nevertheless, the hemispleen liver metastasis model is superior to traditional subcutaneous tumor models, as the tumor microenvironment in the liver metastasis model more closely resembles that of the human PDACs. Our study does not support the combination of IDO1 inhibitor and anti-PD-1/PD-L1 antibodies for PDAC in the presence of vaccine therapy. Although combining anti-PD-1/PD-L1 antibody with IDO1 inhibitor in the presence of vaccine therapy would not result in a synergic effect or a significant additive effect, PDACs that are primed by other mechanisms (e.g., a non-T cell-inflamed mechanism) may still benefit from the combination treatment with IDO1 inhibitor and anti-PD-1/PD-L1 antibody. Therefore, it will be interesting to test whether other treatment modalities such as chemotherapy, radiation therapy, or other immune modulating agents can prime PDACs 
for the combination treatment with IDO1 inhibitor and anti-PD-1/ PD-L1 antibody in the presence or absence of vaccine therapy.

\section{Methods}

Immunohistochemistry. Tumor tissues for human correlative IHC staining were obtained from specimens collected after GVAX vaccine exposure in patients who underwent surgery concurrently at our institution under the Johns Hopkins Medical Institution (JHMI) Institutional Review Board (IRB) approved protocol (NA_00074221) (5). Additional human PDAC specimens treated with neoadjuvant chemotherapy and radiation were also collected under the JHMI IRBapproved protocol (NA_00001584). Formalin-fixed paraffin-embedded (FFPE) tissue blocks were obtained from our pathology archive. All FFPE sections of pancreatic tumor specimens were sectioned at 5 $\mu \mathrm{m}$. IHC staining was performed using Dako Catalyzed Signal Amplification system as previously described (34). Antigen retrieval and IHC staining were performed manually for IDO1 utilizing mouse monoclonal anti-hIDO (4.16H1). Slides were deparaffinized and hydrated in preparation for heat-induced antigen retrieval. Incubation with the primary antibody using optimal conditions was followed by immunostaining development and subsequent $\mathrm{H} \& \mathrm{E}$ counterstain. Slides were then rinsed and mounted with glass coverslips.

All slides were deidentified and scanned, and whole-slide images were individually analyzed using Image Analysis Software (Aperio Technologies). Tumor areas to be analyzed were circled by a pathologist on the H\&E-stained slides in order to include the largest area of continuous neoplastic tissue while excluding normal pancreatic and intestinal tissue. The Positive Pixel Count algorithm was used to quantify IDO1 expression. A value above 350,000 pixels/tumor $\mathrm{mm}^{2}$ was determined to be IDO $1^{\text {hi }}$. Six total samples were identified as IDO1 ${ }^{\text {hi }}$ (Figure 1B). Of these 6, only 3 had tissue available for comprehensive IHC experiments. The Cell Surface Count and Nuclear Count algorithms were used to quantify the number of cells positive for immune cell surface or nuclear markers, respectively. Cell density was defined by the ratio of the positive pixels (excluding the number of weak positive pixels) divided by the total specified tumor area. Automatic quantification results were anonymized and validated first by manual quantification.

Cell lines and media. Panc02 is a highly tumorigenic transplantable PDAC cell line derived from C57BL/6 mice (35). Cells were authenticated by DNA and gene expression profiling and cultured as previously described $(35,36)$. These cells were kept in DMEM media (Life Technologies), 10\% fetal bovine serum (Atlas Biologicals), 1\% L-glutamine (Life Technologies), and 0.5\% penicillin/streptomycin (Life Technologies) at $37^{\circ} \mathrm{C}$ in $10 \% \mathrm{CO}_{2}$. $\mathrm{B} 78 \mathrm{H} 1-\mathrm{GM}$ cells are an MHC class I-negative variant of the B16 melanoma cell line capable of secreting GM-CSF (37). B78H1 cells were maintained in RPMI media (Life Technologies), $10 \%$ fetal bovine serum (Atlas Biologicals), 1\% penicillin/streptomycin (Life Technologies) and 0.5\% L-glutamine (Life Technologies) at $37^{\circ} \mathrm{C}$ in $5 \% \mathrm{CO}_{2}$. Immune analyses were performed using CTL-T cell culture medium consisting of RPMI (Life Technologies), 10\% fetal bovine serum (Atlas Biologicals), 1\% L-glutamine (Life Technologies), $1 \%$ penicillin/streptomycin (Life Technologies), 1\% HEPES (Life Technologies), $1 \%$ nonessential amino acids (Life Technologies), and $0.1 \%$ 2000 times $\beta$-mercaptoethanol (Life Technologies).

Mice and in vivo experiments. Female C57BL/6 mice were purchased from Harlan Laboratories at 7-9 weeks of age and maintained according to the Institutional Animal Care and Use Committee
(IACUC) guidelines. The hemispleen preclinical pancreatic cancer model was performed as previously described utilizing the Panc02 cell line $(8,38)$. In brief, on day 0 the spleen was eviscerated from the mouse, hemisected, and injected with $2 \times 10^{6} \mathrm{Panc} 02$ tumor cells. The injected hemispleen segment was subsequently removed.

IDO1 inhibitor compound was dissolved into Methocel vehicle via 15-minute sonication and given to the tumor-bearing mice by oral gavage twice a day at $200 \mathrm{mg} / \mathrm{kg}$ starting on day 3. As previously described, cyclophosphamide (Cy) (100 mg/kg; Bristol-Myers Squibb) was administered intraperitoneally (i.p.) on day 3 prior to vaccine therapy administration (8). Whole-tumor-cell autologous GVAX immunotherapy was prepared utilizing the cultured Panc02- and GM-CSFexpressing B78H1 cells $(8,39)$. Cells were harvested, washed in PBS, combined at an equal concentration of $2 \times 10^{7} / \mathrm{ml}$, and irradiated at 50 Gy. GVAX was administered subcutaneously in 3 limbs (100 $\mu \mathrm{l} \mathrm{each)} \mathrm{on}$ days 4, 7, 14, and 21. Anti-PD-1 or anti-PD-L1 antibody (5 mg/kg; RMP114; 10F.9G2, BioXcell) or IgG (5 mg/kg; 2A3, BioXcell) were administered i.p. starting on day 3 and continuing twice weekly for a total of 8 doses. Mice were monitored daily for survival analyses and euthanized humanely by $\mathrm{CO}_{2}$ inhalation if the following survival endpoints were met, as determined by IACUC third party management: hunched posture, lethargy, dehydration, significant ascites, and rough hair coat.

Immune analysis of spleen and liver metastasis-infiltrating lymphocytes. Spleen and liver metastasis-infiltrating lymphocytes were analyzed on day 13 following Panc02 tumor inoculation and abbreviated treatment course (Cy, day 3; GVAX, days 4 and 7; IgG, anti-PD-1, or anti-PD-L1 antibody, days 3, 6, and 10; IDO1 inhibitor twice a day starting on day 3). Each liver was mechanically processed sequentially through $40-\mu \mathrm{m}$ and $100-\mu \mathrm{m}$ nylon filters and brought to a volume of $25 \mathrm{ml}$ CTL medium. Each spleen was mechanically processed through two 100- $\mu \mathrm{m}$ nylon filters and brought to a volume of $15 \mathrm{ml} \mathrm{CTL} \mathrm{medi-}$ um. All suspensions were centrifuged at $500 \mathrm{~g}$ for 5 minutes. Liver and spleen cell pellets were suspended in $4 \mathrm{ml} \mathrm{ACK} \mathrm{lysis} \mathrm{(Quality} \mathrm{Bio-}$ logical) for 2 minutes and subsequently spun at $1500 \mathrm{rpm}$ for $5 \mathrm{~min}$ utes. Liver cell pellets were then suspended in $6 \mathrm{ml} 80 \%$ Percoll (GE Healthcare Life Sciences), overlaid with $6 \mathrm{ml} \mathrm{40 \%} \mathrm{Percoll,} \mathrm{and} \mathrm{cen-}$ trifuged at room temperature for 25 minutes at $3200 \mathrm{rpm}$ without the brake. The lymphocyte layer was then removed by an 18-gauge needle and suspended in $10 \mathrm{ml}$ CTL media.

Cell staining and flow cytometry. Following the isolation of tumor infiltrating lymphocytes from murine livers, cells were stained with the Live Dead Aqua Dead Cell Kit (Invitrogen). The lymphocytes were washed and subsequently blocked with mouse Fc antibody (BD Pharmingen) for 10 minutes on ice followed by incubation or staining with cell surface antibodies: CD3-APC (Biolegend), CD4-APC H7 (Biolegend), CD8-PE Cy7 (Biolegend), CD25-BV421 (Biolegend), PD-1-FITC (Biolegend), PD-L1-PE (eBioscience), OX40-PE (Biolegend), CD137-APC (eBioscience), CD45-APC Cy7 (BD Pharmingen), CD11b-PE TR (Life Technologies), Ly6C-PerCP Cy5.5 (eBioscience), Ly6G-V450 (BD Horizon), and $\mathrm{F} 4 / 80-\mathrm{PE} \mathrm{Cy} 7$ (eBioscience) for a 30-minute incubation on ice.

Intracellular antimouse forkhead box P3 (FoxP3) staining was then performed following cell surface cell marker incubation. Lymphocytes were suspended in cold Fix/Perm buffer (eBioscience) and incubated for 30 minutes at $4^{\circ} \mathrm{C}$. The cells were then washed with Perm Buffer (eBioscience). FoxP3-PE (eBioscience) antibody was added and incubated on ice for 30 minutes. Cells were washed with Fix/Perm buffer and assayed on a Gallios flow cytometer (Beckman Coulter). 
For determination of apoptosis in vivo, $\mathrm{CD} 8^{+} \mathrm{T}$ cells from tumors and spleens of treated mice were processed into single-cell suspensions as explained above and cells were stained for cell surface markers including CD3, CD8 and fixable live/dead staining. Cells were then washed twice in FACS buffer and once in $1 \times$ Annexin V Binding Buffer (eBiosciences). For determination of apoptosis cells were incubated for 10 to 15 minutes in $100 \mu \mathrm{l}$ Annexin V binding buffer containing $5 \mu \mathrm{l}$ FITC-conjugated Annexin $\mathrm{V}$ at room temperature and protected from light before assayed on a flow cytometer.

Immune cell isolation and RT-PCR. Isolated liver tumor-infiltrating lymphocytes were enriched for $\mathrm{CD}^{+}$cells using $\mathrm{CD} 8^{-}$isolation kit (Life Technologies) according to protocols provided by the manufacturer. Total mouse MDSCs were isolated by magnetic separation from liver tumor-infiltrating lymphocytes by 2 -step purification using the MDSC isolation kit (Miltenyi Biotec) per the manufacturer's protocol.

TRIzol Reagent (Thermo Fisher Scientific) was used to extract total RNA from tumor-infiltrating lymphocyte cell pellets. The RNA was then converted to cDNA using the Superscript III First Strand Synthesis Supermix Kit (Thermo Fisher Scientific). Quantitative RT-PCR was performed on the StepOnePlus Real Time PCR System (Thermo Fisher Scientific) and analyzed by the StepOne software V2.1. The expression of was measured by SYBR Green-based qPCR. All gene expression was normalized to the expression of $\beta$-actin. All PCR reactions were performed in triplicate.

Mouse IFN- $\gamma$ enzyme-linked immunosorbent assays. $\mathrm{CD}^{+} \mathrm{T}$ cells were isolated from tumor-bearing mice liver and spleen and cocultured with autologous Panc02 tumor cells irradiated with 50 Gy at a ratio of 5:1 $\left(2 \times 10^{5} \mathrm{CD} 8^{+} \mathrm{T}\right.$ cells: $4 \times 10^{4}$ irradiated Panc02 tumor cells). The coculture was incubated for 18 hours in AIMV medium (Thermo Fisher Scientific) at $37^{\circ} \mathrm{C}$. Mouse IFN- $\gamma$ enzyme-linked immunosorbent assay (ELISA) Ready-Set-Go (eBioscience) was then conducted with the supernatant per manufacturer's protocol.

MDSC functional assays. Liver metastases-infiltrating lymphocytes were isolated from tumor-bearing mice and purified by magnetic separation to obtain MDSCs from each treatment group. MDSCs were resuspended in CTL media. $\mathrm{CD}^{+}$cells were isolated from wild-type C57BL/6 mouse splenocytes and stained with the CFSE Cell Proliferation Kit (CellTrace, Thermo Fisher Scientific) per the manufacturer's protocol. Cells were plated in U-bottom 96-well plates at a 2:1 ratio $\left(2 \times 10^{5} \mathrm{CFSE}\right.$ stained CD8: $\left.1 \times 10^{5} \mathrm{MDSCs}\right)$ and incubated at $37^{\circ} \mathrm{C}$ for 48 hours with CD3/CD28 Mouse T-Activator beads (Thermo Fisher Scientific). Cells were then assayed on a Gallios flow cytometer (Beckman Coulter). Mouse Colorimetric Arginase Assay Kit (abcam) was conducted with isolated mouse MDSCs per the manufacturer's protocol.

RNA sequencing and microarray analysis of human PDAC tissue. Approximately twenty 5-mm sections of FFPE tissue from each subject were anonymized and stained with $\mathrm{H} \& \mathrm{E}$ immediately before the stroma were microdissected by a pathologist using a dissecting microscope as previously described (5). RNA was purified using the RecoverAll
Total Nucleic Acid Isolation Kit for FFPE (Ambion) and amplified by the WT-Ovation FFPE System (NuGEN Technologies) as per the manufacturers' protocols. Whole-exome RNA sequencing of dissected pancreatic specimen stroma of the same human cohort of GVAX-treated PDAC patients used for IHC was performed at MEDgenome. The data discussed in this publication have been deposited in the MINSEQEcompliant public NCBI's Gene Expression Omnibus (40) and are accessible through GEO Series accession number GSE125506.

Statistics. All statistical analyses and graphing were performed using GraphPad Prism software (GraphPad Software). Kaplan-Meier curves and log-rank tests were used to estimate median survival and analyze survival outcomes between subgroups. For comparison of cell number, percentage, and cytokine expression, the mean values were evaluated using Student's or Welch's $t$ test. One-way ANOVA was utilized for multiple comparisons of means. A $P$ value of less than 0.05 was considered statistically significant.

Study approval. All studies and maintenance of mice were conducted in accordance with the approval of the Institutional Animal Care and Use Committee (IACUC) guidelines. Tumor tissues for human correlative IHC staining were obtained from specimens collected after GVAX vaccine exposure in patients who underwent surgery concurrently at our institution under the JHMI IRB-approved protocol NA_00074221 (5). Additional human PDAC specimens treated with neoadjuvant chemotherapy and radiation were also collected under the JHMI IRB-approved protocol NA_00001584. Written informed consent was obtained from participants.

\section{Author contributions}

$\mathrm{ABB}, \mathrm{JK}, \mathrm{VK}$, and LZ designed the research studies. ABB, JK, DLT, STM, and VK conducted experiments. ABB, JK, DLT, VK, and LZ acquired data. ABB, JK, DLT, AGM, VK, and LZ analyzed data. $\mathrm{ABB}$ and LZ wrote the manuscript. All authors critically reviewed the manuscript.

\section{Acknowledgments}

This work was supported by a grant from iTeos Therapeutics and also in part by NIH grant R01 CA169702; NIH grant R01 CA197296; NIH grant T32 CA126607; the Viragh Foundation and the Skip Viragh Pancreatic Cancer Center at Johns Hopkins; National Cancer Institute Specialized Programs of Research Excellence in Gastrointestinal Cancers grant P50 CA062924; and Sidney Kimmel Comprehensive Cancer Center grant P30 CA006973. We offer additional sincere appreciation to iTeos Therapeutics for providing the IDO1 inhibitor drug, anti-IDO1 antibody, and IDO1 IHC protocols.

Address correspondence to: Lei Zheng, 1650 Orleans Street, CRB1 Room 488, Baltimore, Maryland 21042, USA. Phone: 410.502.6241; Email: lzheng6@jhmi.edu.
1. Brahmer JR, et al. Safety and activity of antiPD-L1 antibody in patients with advanced cancer. N Engl J Med. 2012;366(26):2455-2465.

2. Blair AB, Zheng L. Rational combinations of immunotherapy for pancreatic ductal adenocarcinoma. Chin Clin Oncol. 2017;6(3):31.

3. Kleponis J, Skelton R, Zheng L. Fueling the engine and releasing the break: combinational therapy of cancer vaccines and immune checkpoint inhibitors. Cancer Biol Med. 2015;12(3):201-208.

4. Foley K, Kim V, Jaffee E, Zheng L. Current progress in immunotherapy for pancreatic cancer. Cancer Lett. 2016;381(1):244-251.

5. Lutz ER, et al. Immunotherapy converts nonim- munogenic pancreatic tumors into immunogenic foci of immune regulation. Cancer Immunol Res. 2014;2(7):616-631.

6. Zheng L. Does vaccine-primed pancreatic cancer offer better candidates for immune-based therapies? Immunotherapy. 2014;6(10):1017-1020.

7. Lutz ER, Kinkead H, Jaffee EM, Zheng L. Priming 
the pancreatic cancer tumor microenvironment for checkpoint-inhibitor immunotherapy. Oncoimmunology. 2014;3(11):e962401.

8. Soares KC, et al. PD-1/PD-L1 blockade together with vaccine therapy facilitates effector T-cell infiltration into pancreatic tumors. JImmunother. 2015;38(1):1-11.

9. Fallarino $\mathrm{F}$, et al. $\mathrm{T}$ cell apoptosis by tryptophan catabolism. Cell Death Differ. 2002;9(10):1069-1077.

10. Fallarino F, et al. The combined effects of tryptophan starvation and tryptophan catabolites down-regulate $\mathrm{T}$ cell receptor zeta-chain and induce a regulatory phenotype in naive $\mathrm{T}$ cells. JImmunol. 2006;176(11):6752-6761.

11. Murphy AG, Zheng L. Small molecule drugs with immunomodulatory effects in cancer. Hum Vaccin Immunother. 2015;11(10):2463-2468.

12. Uyttenhove C, et al. Evidence for a tumoral immune resistance mechanism based on tryptophan degradation by indoleamine 2,3-dioxygenase. Nat Med. 2003;9(10):1269-1274.

13. Smith C, et al. IDO is a nodal pathogenic driver of lung cancer and metastasis development. Cancer Discov. 2012;2(8):722-735.

14. Witkiewicz A, et al. Expression of indoleamine 2,3-dioxygenase in metastatic pancreatic ductal adenocarcinoma recruits regulatory $\mathrm{T}$ cells to avoid immune detection. J Am Coll Surg. 2008;206(5):849-854.

15. Sharma MD, et al. Plasmacytoid dendritic cells from mouse tumor-draining lymph nodes directly activate mature Tregs via indoleamine 2,3-dioxygenase. JClin Invest. 2007;117(9):2570-2582.

16. Munn DH, et al. Expression of indoleamine 2,3-dioxygenase by plasmacytoid dendritic cells in tumor-draining lymph nodes. J Clin Invest. 2004;114(2):280-290.

17. Munn DH, Mellor AL. Indoleamine 2,3-dioxygenase and tumor-induced tolerance. JClin Invest. 2007;117(5):1147-1154.

18. Balachandran VP, et al. Imatinib potentiates antitumor $\mathrm{T}$ cell responses in gastrointestinal stromal tumor through the inhibition of Ido. Nat Med. 2011;17(9):1094-1100.
19. Yu J, et al. Myeloid-derived suppressor cells suppress antitumor immune responses through IDO expression and correlate with lymph node metastasis in patients with breast cancer. JImmunol. 2013;190(7):3783-3797.

20. Beatty GL, et al. First-in-human phase I study of the oral inhibitor of indoleamine 2,3-dioxygenase-1 epacadostat (INCB024360) in patients with advanced solid malignancies. Clin Cancer Res. 2017;23(13):3269-3276.

21. Gibney GT, et al. Preliminary results from a phase $1 / 2$ study of INCB024360 combined with ipilimumab (ipi) in patients (pts) with melanoma. JClin Oncol. 2014;32(15_suppl):3010.

22. Hamid O, et al. Epacadostat plus pembrolizumab in patients with advanced melanoma: Phase 1 and 2 efficacy and safety results from ECHO-202/KEYNOTE-037. Annals of Oncology. 2017;28(suppl_5):v428-v448.

23. Long GV, et al. Epacadostat plus pembrolizumab versus pembrolizumab alone in patients with unresectable or metastatic melanoma: Results of the phase 3 ECHO-301/KEYNOTE-252 study. JClin Oncol. 2018;36(15_suppl):108.

24. Yasui H, Takai K, Yoshida R, Hayaishi O. Interferon enhances tryptophan metabolism by inducing pulmonary indoleamine 2,3-dioxygenase: its possible occurrence in cancer patients. Proc Natl Acad Sci U S A. 1986;83(17):6622-6626.

25. Jung ID, et al. Differential regulation of indoleamine 2,3-dioxygenase by lipopolysaccharide and interferon gamma in murine bone marrow derived dendritic cells. FEBS Lett. 2007;581(7):1449-1456.

26. Tsujikawa T, et al. Quantitative multiplex immunohistochemistry reveals myeloid-inflamed tumor-immune complexity associated with poor prognosis. Cell Rep. 2017;19(1):203-217.

27. Basdeo SA, et al. Ex-Th17 (nonclassical Th1) cells are functionally distinct from classical $\mathrm{Th} 1$ and Th17 cells and are not constrained by regulatory T cells. J Immunol. 2017;198(6):2249-2259.

28. Bailey SR, Nelson MH, Himes RA, Li Z, Mehrotra S, Paulos CM. Th17 cells in cancer: the ultimate identity crisis. Front Immunol. 2014;5:276.

29. Wang L, Yi T, Kortylewski M, Pardoll DM,
Zeng D, Yu H. IL-17 can promote tumor growth through an IL-6-Stat3 signaling pathway. J Exp Med.2009;206(7):1457-1464.

30. Hirota K, et al. Fate mapping of IL-17-producing $\mathrm{T}$ cells in inflammatory responses. Nat Immunol. 2011;12(3):255-263.

31. Lee YK, Mukasa R, Hatton RD, Weaver CT. Developmental plasticity of Th17 and Treg cells. Curr Opin Immunol. 2009;21(3):274-280.

32. Jin W, Dong C. IL-17 cytokines in immunity and inflammation. Emerg Microbes Infect. 2013;2(9):e60.

33. Shrimali RK, et al. Concurrent PD-1 blockade negates the effects of OX40 agonist antibody in combination immunotherapy through inducing T-cell apoptosis. Cancer Immunol Res. 2017;5(9):755-766.

34. Bigelow E, et al. Immunohistochemical staining of B7-H1 (PD-L1) on paraffin-embedded slides of pancreatic adenocarcinoma tissue. JVis Exp. 2013;(71): e4059.

35. Corbett TH, et al. Induction and chemotherapeutic response of two transplantable ductal adenocarcinomas of the pancreas in C57BL/6 mice. Cancer Res. 1984;44(2):717-726.

36. Rucki AA, et al. Heterogeneous stromal signaling within the tumor microenvironment controls the metastasis of pancreatic cancer. Cancer Res. 2017;77(1):41-52.

37. Levitsky HI, Lazenby A, Hayashi RJ, Pardoll DM. In vivo priming of two distinct antitumor effector populations: the role of MHC class I expression. JExp Med.1994;179(4):1215-1224.

38. Soares KC, et al. A preclinical murine model of hepatic metastases. J Vis Exp. 2014;(91):51677.

39. Leao IC, Ganesan P, Armstrong TD, Jaffee EM. Effective depletion of regulatory $\mathrm{T}$ cells allows the recruitment of mesothelin-specific CD8 T cells to the antitumor immune response against a mesothelin-expressing mouse pancreatic adenocarcinoma. Clin Transl Sci. 2008;1(3):228-239.

40. Edgar R, Domrachev M, Lash AE. Gene Expression Omnibus: NCBI gene expression and hybridization array data repository. Nucleic Acids Res. 2002;30(1):207-210. 